\title{
Comparativo de las Propiedades Psicométricas del NAQ-R y del LIPT-60 en trabajadores venezolanos
}

\section{Comparison of Psychometric Properties of NAQ-R and LIPT-60 in a Sample of Venezuelan Workers}

\author{
Anthony Millán ${ }^{1 \text { ORCID }}$, Luciana Diaferia² - Marian Acosta ${ }^{3}$ - María Eugenia D’Aubeterre ${ }^{4}$ ORCID \\ 1,2,3 Universidad Metropolitana, ${ }^{4}$ Universidad Central de Venezuela
}

Venezuela

Fecha correspondencia:

Recibido: agosto 28 de 2015.

Aceptado: junio 7 de 2016.

\section{Forma de citar:}

Millán, A., Diaferia, L., Acosta,

M., \& D'Aubeterre, M.E. (2016).

Comparativo de las Propiedades

Psicométricas del NAQ-R y del LIPT-

60 en trabajadores venezolanos.

Rev. CES Psicol., 9(2),40-67.

\section{Open access}

(c) Copyright

Licencia creative commons

Etica de publicaciones

Revisión por pares

Gestión por Open Journal System

ISSN: 2011-3080

DOI: http://dx.doi.org/10.21615/

cesp.9.2.4

Sobre los autores:

1. Doctorando en Ciencias Sociales y Humanidades de la Universidad Simón Bolívar, Venezuela.

Docente Asociado, Departamento de Ciencias del Comportamiento Escuela de Psicología, Universidad Metropolitana (UNIMET), Venezuela.

Comparte

\section{Resumen}

El acoso laboral [mobbing] es un tipo de intimidación dentro del trabajo que, desde un punto de vista psicométrico, no puede medirse directamente. La utilización de escalas como Mobbing-UNIPSICO Scale, NAQ, LIPT, HPT, EAPA-T, CISNEROS, entre otras, busca predecir de forma indirecta su impacto en la salud de los trabajadores. En Venezuela existe una validación del LIPT (versión de 60 ítems), que presenta defectos metodológicos. Por ello se re-evaluó sus propiedades psicométricas y se compararon con las del NAQ (versión R), que es el otro instrumento más comúnmente utilizado para medir acoso laboral (de tipo vertical), para así determinar cuál es más pertinente para emitir un diagnóstico. Si bien el resultado de la validez de constructo señala que hay un mejor ajuste en la estructura factorial del $N A Q-R$, se concluye que una versión más corta del LIPT (de 45 ítems) es más precisa para emitir un diagnóstico, dado que predice mejor los distintos indicadores de salud psicológica en el trabajo y además posee mejores indicadores de consistencia interna.

Palabras claves: Acoso Laboral, Intimidación, Validación, Test Psicométrico.

\section{Abstract}

Mobbing is a type of intimidation into the workplace, and it cannot be directly measured from a psychometric view. The administration of different scales aims indirectly to predict their impact on workers' health. These scales are: Mobbing-UNIPSICO Scale, NAQ, LIPT, HPT, EAPA-T, CISNEROS, among others; The LIPT-60 (60-item version) is used in Venezuela, but due to the fact this scale has presented methodological flaws, its psychometric properties were re-evaluated and compared with those of the NAQ (R version), another instrument commonly used to measure mobbing (vertical type), in order to determine which of these scales is more reliable to make a diagnosis. While the outcome of the construct validity indicates that there is a better fit in the factorial structure of the NAQ-R, we concluded that a shorter version of LIPT ( 45 items) is more accurate to make a diagnosis, since it predicts better the various indicators of psychological health at work and also presents better indicators of internal consistency.

Keywords: Bullying, Mobbing, Validation, Test Validity, Psychometric Test 
Pág 41

2. Universidad Metropolitana.

3. Universidad Metropolitana.

4. Doctorado en Antropología, Escuela Nacional de Antropología e Historia, México. Maestría en Ciencias Sociales, Universidad Autónoma de Puebla, México, Licenciatura en Psicología Social, Universidad Central de Venezuela.

\section{Introducción}

El acoso laboral [mobbing] es un tipo de hostigamiento recurrente y prolongado hacia una o más personas dentro del lugar de trabajo. De acuerdo con Einarsen, Hoel, Zapf y Cooper (2003), significa "acosar, ofender, excluir socialmente o afectar negativamente la labor de alguien. Es un proceso de aumento progresivo en cuyo curso la persona confrontada termina colocada en una posición inferior y se convierte en el blanco de actos sociales negativos sistemáticos" (p.15). De acuerdo con Kahale (2015), el término también es conocido como:

Acoso moral, acoso laboral, acoso psicológico en el trabajo, psicoterror laboral, hostigamiento laboral, asesinato psicológico y hostigamiento psicológico en el trabajo. No obstante, además de las anteriores expresiones, a nivel jurisprudencial, existen otras menos frecuentes como violencia psicológica, proceso de distracción, acoso ambiental, orquestación de la creación de un ambiente hostil y feudalismo industrial (p.24).

A nivel internacional, la Organización Mundial de la Salud [OMS], en el año 2010, lo consideró como un factor de riesgo psicosocial que repercute tanto en la salud física como en la psicológica del trabajador. En Venezuela también se reconoce su existencia, tanto en el Artículo 164 de la Ley Orgánica del Trabajo, Las Trabajadoras y los Trabajadores [LOTTT] del año 2012, como en el Baremo Nacional para la Asignación de Porcentaje de Discapacidad por Enfermedades Ocupacionales y Accidentes de Trabajo del año 2013, establecido por el Instituto Nacional de Prevención, Salud y Seguridad Laborales [INPSASEL] y en la Norma Técnica para la Declaración de Enfermedad Ocupacional [NT-02-2008], establecido también por el INPSASEL en el año 2008. Esto corresponde de manera coherente con los artículos 129 y 130 de la Ley Orgánica de Prevención, Condiciones y Medio Ambiente de Trabajo [LOPCYMAT] del año 2005. Sin embargo, existe aún cierta imprecisión en lo que respecta a sus características específicas, lo cual ha traído como consecuencia que los instrumentos psicológicos que lo registran tengan un número diferente de comportamientos asociados a ella, pues muchos de estos instrumentos suponen simplemente una lista de cotejo que está construida por el consenso de un grupo de expertos, pero sin criterios psicométricos que los avalen empíricamente (Escartín, Rodríquez-Carballeira, Gómez-Benito, \& Zapf, 2010).

A este respecto, Kahale (2015) señala que la ausencia de una terminología unificadora para definir al acoso laboral ha traído las siguientes consecuencias: a) la ausencia de una delimitación de la conducta jurídicamente relevante; b) la falta de delimitación del derecho fundamental vulnerado de forma específica por la conducta del hostigador, y c) la dificultad de seleccionar la técnica reguladora más idónea para la tutela judicial efectiva del derecho del lesionado, así como la fijación de las consecuencias jurídicas derivadas de la conducta ilícita.

En este sentido, si bien es cierto que en el año 2002 la Organización Internacional del Trabajo [OIT] consideró perentorio tipificar al acoso laboral como una enfermedad ocupacional, oficializando su inclusión en el año 2005 en la lista de enfermedades laborales, en el año 2009 fue retirada hasta tanto no se establezca, científicamente o por métodos adecuados a las condiciones y la práctica nacionales, un vínculo directo entre la exposición a factores de riesgo que resulte de las actividades laborales y el(los) trastorno(s) mentales o del comportamiento contraído(s) por el trabajador (OIT, 2010). 
Pág 42

"Llama la atención que en la gran mayoría [de las sentencias] el trabajador ha fracasado en su pretensión; es decir el acosado no ha visto reconocido, o no ha podido demostrar el hostigamiento laboral sufrido en la empresa. Por consecuencia, existe un desconocimiento de qué se entiende por acoso laboral y cuáles son las vías idóneas para erradicarlo" (Kahale, 2015, p. 170).
En el caso venezolano, ni el Baremo Nacional para la Asignación de Porcentaje de Discapacidad por Enfermedades Ocupacionales y Accidentes de Trabajo (INPSASEL, 2013), ni la NT-02-2008, aclaran este asunto, dejando en manos del psicólogo o psiquiatra que establece el diagnóstico la obligación de esclarecerlo en su respectivo informe. No obstante, en la actualidad la selección de la estrategia de evaluación que hace el perito debe cumplir con el más alto estándar científico (Devis-Echandía, 1993), de manera de asegurarle al imputado el respeto a su presunción de inocencia, el cual es un derecho humano, consagrado en la Declaración Universal de los Derechos Humanos, y en la Constitución de la República Bolivariana de Venezuela (1999) y el artículo 8 del Código Orgánico Procesal Penal (2012). Por ésta razón existe el proceso de apreciación o valoración de pruebas, que "si bien lo debe realizar el juez o el tribunal al momento de decidir, también está precedido por la actividad crítica que de las pruebas hacen las partes, colaborando de esa manera con el sentenciador" (Delgado, 2015, p. 97); en éste sentido, el peritaje no es vinculante, y debe ser apreciado como una prueba más, individualmente y dentro del conjunto probatorio general. A lo anterior se suma lo que señala Kahale (2015) para el caso del mobbing: "llama la atención que en la gran mayoría [de las sentencias] el trabajador ha fracasado en su pretensión; es decir el acosado no ha visto reconocido, o no ha podido demostrar el hostigamiento laboral sufrido en la empresa. Por consecuencia, existe un desconocimiento de qué se entiende por acoso laboral y cuáles son las vías idóneas para erradicarlo" (Kahale, 2015, p. 170).

Así, resulta evidente la necesidad de establecer estudios científicos relacionados con la calidad técnica de los instrumentos que miden el acoso psicológico en el trabajo. En lo que respecta al presente trabajo de investigación, el esfuerzo fundamental consistió en analizar y comparar estas características en dos de los instrumentos más utilizados a nivel internacional para medir acoso laboral de tipo vertical, que, de acuerdo con Kahale (2015), es el que sucede desde un jefe hacia sus subordinados o personas que poseen un cargo de jerarquía inferior, dada la percepción del jefe de una amenaza a su capacidad de control sobre el grupo. Los instrumentos de medición estudiados son el Leymann Inventory of Psychological Terrorization [LIPT, por sus siglas en inglés] y el Negative Acts Questionnaire [NAQ, por sus siglas en inglés]. La intención del presente estudio es determinar cuál de ellos es el más pertinente desde un punto de vista empírico para evaluar dicho fenómeno, con lo cual se brinda información técnica relevante a los psicólogos y psiquiatras que requieran formular diagnósticos en esta materia con el respaldo científico adecuado.

Asimismo, es importante destacar que este estudio se inscribe en la línea de investigación de salud psicológica del Departamento de Ciencias del Comportamiento de la Universidad Metropolitana en Venezuela; se inserta también dentro de la necesidad de investigación $N^{\circ} 343$, relativa a la línea estratégica de aspectos sociales, culturales, psicológicos y genéticos asociados a la salud laboral, establecida por el Ministerio del Poder Popular para Ciencia, Tecnología e Innovación [MCTI] de Venezuela en el año 2011, y a la necesidad N³84, que plantea la prevalencia, prevención, caracterización y alternativas terapéuticas de enfermedades crónicas en contextos laborales (MCTI, 2011).

\section{Medición del acoso psicológico en el trabajo}

Kahale (2015) señala que el estudio del acoso psicológico en el trabajo comienza con los estudios de Leymann (1990), quien elabora un cuestionario con 45 ítems agrupados en cinco factores que describen actividades características del acoso laboral, denominado Leymann Inventory of Psychological Terrorization [LIPT, por sus siglas en inglés]. + 
El LIPT ha sido revisado por diversos autores como Niedl (1996) y Zapf, Knorz y Kulla (1996) en Alemania; Vartia (1996) en Finlandia; Ege (2002) en Italia, y González de Rivera y Rodríguez-Abuín (2003, 2005) en España. Si bien en la mayoría de los casos se produce únicamente una traducción y adaptación de los ítems a la población, en otros se emplean versiones reducidas o ampliadas de la escala. Además, en algunas ocasiones la revisión viene acompañada de un análisis factorial (Niedl, 1996; Zapf, 1999; Zapf et al., 1996). Específicamente, González y Rodríquez (2003) tradujeron, adaptaron y modificaron las posibilidades de respuesta de dicotómica a escala Likert, además de agregarle 15 nuevos ítems, dando origen al LIPT-60. Aunque en este último se describe el procedimiento para su puntuación y algunas comparaciones y correlaciones del estudio con variables sociodemográficas, este no presenta indicadores de fiabilidad y validez que avale psicométricamente dicha modificación con respecto a la versión original.

Otro de los instrumentos más usados es el Negative Acts Questionnaire [NAQ, por sus siglas en inglés], \pm elaborado en Noruega por Einarsen y Raknes (1997), compuesto por 23 conductas negativas en el ambiente de trabajo, agrupadas en 3 factores, con una confiabilidad de 0.92 .

Esta versión ha sido empleada en varios estudios (Einarsen Matthiesen, \& Skogstad, 1998; Matthiesen \& Einarsen, 2001; Mikkelsen \& Einarsen, 2001), aunque también lo ha sido introduciendo ciertas modificaciones como la escala de 27 ítems (Matthiesen, Einarsen, \& Mykletun, 2008), la de 28 ítems (Glaso, Matthiesen, Nielsen, \& Einarsen, 2007), la de 18 (Matthiesen \& Einarsen, 2007; Mikkelsen \& Einarsen, 2002), la de 17 (Giorgi, Matthiesen, \& Einarsen, 2006), la de 16 (Notelaers, Einarsen, De Witte, \& Vermunt, 2006), la de 14 ítems (Moreno-Jiménez, Rodríguez-Muñoz, Martínez-Gamarra, \& Gálvez, 2007) y el NAQ-R de 22 ítems (Einarsen \& Hoel, 2006; Hauge, Skogstad, \& Einarsen, 2007; Nielsen \& Einarsen, 2008), en el que la consistencia interna ha oscilado entre 0.83 y 0.96.

Este cuestionario, en sus diferentes versiones, también ha sido adaptado a las características laborales de diferentes países, hallándose una estructura bifactorial (Einarsen \& Hoel, 2001; Einarsen Hoel, \& Notelaers, 2009; Hoel, Cooper, \& Faragher, 2001; Hoel, Faragher, \& Cooper, 2004), 3 factores (Einarsen et al., 2009), 4 factores (Hoel \& Cooper, 2000a) y ha sido adaptado al francés, flamenco (Notelaers et al., 2006) y al italiano (Giorgi, et al., 2006).

Al igual que el LIPT, el NAQ también ha sido objeto de traducción y revisión en España, país que lleva la delantera en investigaciones sobre acoso laboral. Cabe recordar que el primer trabajo de adaptación lo realizaron García-Izquierdo, Saéz y Llor (2003), cuyos resultados indicaron que la versión de 22 ítems tiene una buena consistencia interna ( $\alpha=.85)$, siete factores que explican el $66,7 \%$ de la varianza y correlaciones significativamente elevadas con diversos criterios externos: satisfacción laboral, agotamiento emocional, cinismo y eficacia profesional. Luego, los investigadores mencionados y otros realizaron una revisión (NAQ-RE) en la que obtuvieron una escala de 24 ítems (García-Izquierdo et al., 2004) que arrojó una estructura factorial de cuatro factores que explican el $56,10 \%$ de la varianza, con un $\alpha=.91$ y correlaciones significativamente elevadas con la satisfacción laboral, con el burnout (agotamiento emocional y cinismo) y con ciertas variables relacionadas con la salud.

Posteriormente, González y Graña (2009) evaluaron las propiedades psicométricas de la versión española del NAQ-R y señalaron que el mismo cuenta con las suficientes propiedades psicométricas para medir eficazmente el constructo. 
Pág 44

Tanto el LIPT como el NAQR son ejemplos de lo que León-Pérez et al. (2004), denominan como métodos de auto-etiquetado [SLM, por sus siglas en inglés: self-labeling method], los cuales se caracterizan por partir de la percepción del encuestado.
Más recientemente, Gil-Monte, Carretero y Luciano (2006) crearon un nuevo cuestionario denominado Mobbing-UNIPSICO Scale, \pm que es una adaptación de 20 ítems provenientes tanto del LIPT como del NAQ, y ha sido utilizado para evaluar a empleados que trabajan con personas con discapacidad intelectual (Figueiredo-Ferraz, Gil-Monte, Grau-Alberola, Llorca-Pellicer, \& García-Juesas, 2012; Figueiredo-Ferraz, Gil-Monte, \& Olivares-Faúndez, 2015). Así mismo, estudios como el de León-Pérez, Notelaers, Arenas, Munduate y Medina (2014) han abordado el estudio del acoso laboral desde una perspectiva metodológica distinta a la anteriormente señalada, la cual se enfoca en la descripción de perfiles de acoso. Estos autores lo abordan desde la construcción de tipologías de trabajadores con diferencias en cuanto a la naturaleza e intensidad de los comportamientos de intimidación registrados a partir del NAQ.

Finalmente, también han aparecido otros instrumentos que miden el acoso psicológico en el trabajo, de forma independiente al desarrollo del NAQ y al LIPT, como el Cuestionario de Hostigamiento Psicológico en el Trabajo [HPT], de Fornés, MartínezAbascal y García (2008), el cual se circunscribe específicamente al ámbito de la enfermería, o la Escala de Abuso Psicológico en el Lugar de Trabajo [EAPA-T] de Escartín et al. (2010), que intenta ser un instrumento de medición corto y culturalmente válido para el acoso laboral en España. Otro instrumento comúnmente citado en la bibliografía académica es la Escala CISNEROS de Piñuel (2001), componente del barómetro CISNEROS $^{\circledR} \pm$ que, de acuerdo con Fidalgo \& Piñuel (2004), fue la primera herramienta que se utilizó en España para medir la incidencia del fenómeno del acoso psicológico. Esta ha sido utilizada en Venezuela para evaluar la existencia de mobbing en los trabajadores de una Institución de Salud Pública (Cardozo \& López, 2009), sin la existencia de una validación previa para éste país. Además, incorpora en su medición la evaluación del acoso entre compañeros de trabajo o de tipo horizontal (Kahale, 2015), no permitiría la comparación con los otros instrumentos considerados (el LIPT y el NAQ) que se concentran solo en el acoso de tipo vertical.

Por otra parte, tanto el LIPT como el NAQR son ejemplos de lo que León-Pérez et al. (2004), denominan como métodos de auto-etiquetado [SLM, por sus siglas en inglés: self-labeling method], los cuales se caracterizan por partir de la percepción del encuestado. Estos autores comentan que, si bien este método es fácil de administrar y su validez aparente es convincente, también ha sido criticado por adoptar un enfoque subjetivo en el que la personalidad, factores emocionales, factores cognitivos y percepciones erróneas pueden afectar o sesgar la medición; además de no proporcionar ninguna información sobre la naturaleza de los comportamientos que intervienen en la situación de acoso. Finalmente, en términos metodológicos, éstos autores señalan que han sido criticados porque, al establecer la prevalencia del acoso, este enfoque utiliza puntos de corte arbitrarios para distinguir entre aquellos que sí fueron acosados de los que no fueron no acosados. De hecho, señalan que estos aspectos podrían explicar la alta disparidad en la tasa de prevalencia del acoso laboral cuando estos inventarios se han aplicado a través de diferentes países y también dentro de un mismo país.

Una manera de resolver estos problemas es el uso de indicadores de salud psicológica ocupacional como criterios externos, lo que permitiría verificar, independientemente de la naturaleza subjetiva de las medidas SLM, si dicha percepción guarda algún tipo de correlato con su salud a nivel psicológico. De esta forma, si bien el puntaje obtenido a través de estos mecanismos no determina si hubo o no acoso en el trabajo, el procedimiento sí permitiría estimar el efecto que tendrían estos indi- 
cadores sobre la salud como una evidencia indirecta de los mismos. Ejemplo de lo anterior han sido los estudios de León-Pérez et al. (2004), Mayhew y Chappell (2007) o Escartín et al. (2010), quienes utilizaron como criterios externos a los test de acoso psicológico en el trabajo: el bienestar psicológico, los trastornos de estrés postraumático, la ansiedad, la depresión, entre otros constructos. En resumen, este enfoque permitiría un abordaje indirecto del acoso psicológico con miras a una evaluación más bien preventiva, sin dejar de ser crucial en el esclarecimiento de la relación existente entre la exposición a conductas de intimidación y la salud (Vie, Glaso, \& Einarsen, 2011).

En Venezuela, el acoso laboral es un tema poco estudiado y sólo se conoce la validación del LIPT-60 (Jiménez, 2010), que arroja una confiabilidad de 98 pero presenta algunas fallas psicométricas: tamaño muestral pequeño $(n=209)$, confunde el término de validez de constructo con el de confiabilidad y asume la existencia de una unidimensionalidad en una prueba factorial sin realizar esfuerzos de cálculo específicos para comprobar la veracidad de tal hipótesis. Tampoco hay consistencia en la descripción del tamaño de la muestra a lo largo del documento y, además, el estudio posee el sesgo de indeterminación factorial y confirmatorio para determinar la posible existencia de una estructura factorial subyacente (Millán, Calvanese, \& D'Aubeterre, 2013) y solo presenta la medida de la adecuación muestral de Kaiser, Meyer y Olkin [KMO], por lo que no puede demostrarse la presencia de una estructura factorial subyacente. Tal enfoque posee también el sesgo de capitalización del azar, pues no verifica el resultado con una segunda muestra o por medio del Bootstraping, realiza primero la validez predictiva y luego la de constructo, no presenta la estructura factorial obtenida, no declara el método utilizado para su determinación y el número de factores obtenidos (11) no coincide con el número de factores teóricos; por lo que pareciera, de acuerdo con éstos resultados, que la prueba no tiene validez de constructo, y no propone ninguna explicación al respecto. Finalmente, lo que declara como análisis factorial confirmatorio es un segundo análisis factorial exploratorio que, en vez de correrse sobre los ítems, se corre sobre el puntaje en los factores y de este segundo Análisis Factorial Exploratorio [AFE], no se reporta ningún indicador que demuestre su adecuación.

Estas características no son distintas a las observadas en otros estudios de validación del LIPT, pues como señala Escartín et al. (2010), al validar el LIPT-60, no se llevó a cabo ningún análisis factorial confirmatorio y los elementos agregados a la versión original no representaban una categoría homogénea. De hecho, la adición de estos 15 ítems a la versión original del LIPT no sucedió como consecuencia de evidencias empíricas que lo justificaran.

Según lo descrito anteriormente, y debido a la variedad de adaptaciones, traducciones, revisiones y validaciones que arrojan diferentes estructuras factoriales y falta de consenso entre los autores, se justifica metodológicamente la realización de la presente investigación que tuvo como objetivo la evaluación de las propiedades psicométricas del NAQ-R y el LIPT-60, con el fin de determinar cuál de estas presentaba mejores indicadores psicométricos tanto a nivel de validez de constructo como de criterio. Como señala Moayed, Daraiseh, Shell y Salem (2006) queda la posibilidad de que exista una superposición entre todas estas escalas, lo cual avalaría el uso de cualquiera de ellos, dado que ambos instrumentos, aunque diferentes en apariencia, llegarían a la misma conclusión en el diagnóstico. No obstante, también existe la posibilidad de lo contrario y la decisión de selección del instrumento no debería realizarse a partir de criterios distintos a los meramente técnicos. 
Pág 46

Específicamente, el objetivo es identificar y confirmar las diversas estructuras factoriales del NAQ-R y LIPT-60 en una muestra multiocupacional de trabajadores venezolanos (validez de constructo), así como evaluar la validez predictiva que tienen cada uno de ellos con respecto a indicadores de salud psicológica ocupacional, a saber, burnout como indicador legalmente instituido de enfermedad o afección por factores psicosociales en el trabajo (NT-02-2008) sancionadas por el INPSASEL (2013), y con el bienestar psicológico como indicador de salud mental más allá de la enfermedad (World Health Organization [WHO], 1946; Ley Orgánica de la Salud [LOS],1998).

\section{Método}

De acuerdo con Montero y León (2007), el presente es un estudio cuantitativo e instrumental, ya que está encaminado al estudio de las propiedades psicométricas del NAQ-R y LIPT-60. Específicamente, el objetivo es identificar y confirmar las diversas estructuras factoriales del NAQ-R y LIPT-60 en una muestra multiocupacional de trabajadores venezolanos (validez de constructo), así como evaluar la validez predictiva que tienen cada uno de ellos con respecto a indicadores de salud psicológica ocupacional, a saber, burnout como indicador legalmente instituido de enfermedad o afección por factores psicosociales en el trabajo (NT-02-2008) sancionadas por el INPSASEL (2013), y con el bienestar psicológico como indicador de salud mental más allá de la enfermedad (World Health Organization [WHO], 1946; Ley Orgánica de la Salud [LOS],1998).

De hecho, Nunally y Bernstein (1995) señalan al respecto que:

Los tribunales demandan cada vez más alguna conexión lógica entre el predictor y el criterio (es decir, la validez de constructo). El papel de la teoría al guiar la predicción es consistente con el punto de vista de que toda validez es validez de constructo, pero no debemos perder el punto de que la validez predictiva está vinculada con un criterio relativamente específico, a diferencia de la validez de constructo (p. 106).

En este sentido, la direccionalidad esperada entre los coeficientes betas [ $\beta$ ] tanto del NAQ como del LIPT con respecto al Burnout es directa [+], en tanto que se espera teóricamente que a medida que aumentan los puntajes en estos instrumentos, aumente concomitantemente el grado de presencia del Burnout. Por el contrario, con respecto al bienestar psicológico, se espera que dicha direccionalidad sea inversa [-], pues a mayor percepción de acoso psicológico en el trabajo menor bienestar psicológico del trabajador.

El proceso supuso la transcripción de datos, el análisis exploratorio de datos [EDA], el análisis de ítems y el análisis factorial en dos fases descrito por Anderson y Gerbin (1988), Boomsma (2000) y más recientemente por Millán et al (2013). Este método de validación de constructo busca solventar el error de indeterminación factorial del AFE descrito por Pérez-Gil, Chacón y Moreno (2000) y el sesgo confirmatorio del Análisis Factorial Confirmatorio (AFC) (Hair Anderson, Tatham, \& Black, 1999) al evaluar el nivel y grado de ajuste específico de cada uno de los modelos factoriales posibles y compararlos entre sí para descartar aquellos con el menor ajuste a los datos, procedimiento coherente con los estándares internacionales de validación de test psicométricos (Prieto \& Muñiz, 2000; Borsboom, 2006; Ponsoda, 2009). Adicionalmente, el AFC permite darle un carácter causal a la medición del constructo (Batista-Foguet, Coenders, \& Alonso, 2004) como variable latente [0], entre los ítems concebidos como estímulos [E] y las respuestas de cada sujeto a cada uno de ellos [R], brindándole así una mayor validez de constructo a dicha medida. El diseño de investigación es confirmatorio (Prieto \& Muñiz, 2000) no sólo porque se analizan las estructuras factoriales obtenidas en la primera fase con el AFE, sino también porque permite evaluar el ajuste de las estructuras factoriales obtenidas en los estudios previos. La selección de los participantes se realizó con apoyo logístico de los estudiantes de la asignatura de Psicometría I de la Escuela de Psicología de la Universidad Metropolitana (UNIMET) en Caracas. Previo entrenamiento para su aplicación según los lineamientos y como parte del modelo de aprendizaje por proyectos que posee el Diseño Instruccional (DIUM) de dicha universidad. 


\section{Participantes:}

La muestra fue de tipo no probabilística debido a la participación voluntaria y no aleatoria en la misma. Además, fue propositivo o por conveniencia (Hernández, Fernández, \& Baptista, 2007, p.241), ya que para seleccionar a los participantes se utilizaron criterios específicos acordes con el interés del estudio; por ejemplo, que se encontraran desempeñando en la actualidad una actividad del sector formal de la economía venezolana, y se aseguró el balanceo estadístico en cuanto al sector económico en el que laboraban (público=49.52\%; privado $=50.48 \% ; \chi^{2}=0.153 ; p=0.695$ ) y el sexo (femenino $=51 \%$; masculino $\left.=49 \% ; \chi^{2}=0.905 ; p=0.341\right)$, mediante la técnica de control por apareamiento al momento de la selección (Arnau, 1979), concebida para maximizar la heterogeneidad de la muestra y captar así la mayor diversidad de valoraciones en torno al acoso psicológico en el trabajo.

Además, se contó con la presencia de varias profesiones y cargos: personal estratégico (como gerentes y supervisores), profesionales libres (como psicólogos, médicos y abogados), personal administrativo, auxiliar y de apoyo, personal de seguridad, obreros y constructores, vendedores, comerciantes y otros.

\section{Instrumentos}

Negative Acts Questionnaire Revised (NAQ-R). El NAQ-R es un cuestionario que mide las conductas hostiles, con la ventaja de que las afirmaciones a evaluar no plantean explícitamente que están vinculadas al Acoso Laboral, permitiendo así que el encuestado no se ubique en alguno de los roles (acosado o acosador), lo que posibilita la medición de la percepción de acoso más no pretende diagnosticar dicho constructo (González \& Graña, 2009).

La consigna del cuestionario solicita dar respuesta a la pregunta sobre la frecuencia con que en los últimos seis meses se ha producido cada uno de los 24 «actos negativos» en el trabajo que se señalan. La misma cuenta con cinco posibles respuestas, siendo una escala tipo Likert, donde 1 es nunca, a veces es 2, mensualmente es 3 , semanalmente es 4 y diariamente es 5 .

Los instrumentos que se emplearon en esta investigación fueron traducidos y validados con anterioridad. Autores como González \& Graña (2009), recurriendo a realizar la traducción del NAQ apoyados en la técnica llamada back translation. El NAQ-R entonces habría sido traducido al castellano por dos expertos en este ámbito, para luego volver a realizar el proceso pero ahora al inglés por otros dos expertos diferentes, con el fin obtener una versión final (la empleada en este estudio).

Leymann Inventory of Psychological Terrorization (LIPT-60). El LIPT-60 es un cuestionario escalar y autoadministrado que objetiva y valora 60 diferentes estrategias de acoso psicológico a través de una escala Likert que mide la intensidad con que cada una de las conductas de acoso ha afectado al sujeto, desde cero (la conducta no ha tenido lugar) hasta cuatro (conducta de intensidad máxima).

González de Rivera y Rodríguez-Abuín $(2003,2005)$ proponen que, para corregir el LIPT, se establecen tres parámetros globales: el número total de estrategias de acoso psicológico [NEAP], el índice global de acoso psicológico [IGAP] y el índice medio de acoso psicológico [IMAP]. El cálculo de estos parámetros globales se efectúa de la manera siguiente: 
NEAP: Conteo simple de todas las respuestas distintas de cero. Esta medida es conceptualmente la misma que el LIPT total calculado con el cuestionario dicotómico, aunque presenta diferencias de orden metodológico que después discutiremos.

IGAP: Índice global, obtenido sumando los valores asignados a cada estrategia de acoso psicológico y dividiendo esta suma entre el número total de estrategias consideradas en el cuestionario, es decir, entre 60.

IMAP: Índice medio de intensidad de las estrategias de acoso psicológico experimentadas, obtenido dividiendo la suma de los valores asignados a cada estrategia entre el número total de respuestas positivas. Este número es variable y viene determinado por el NEAP: IMAP= Suma de la intensidad de cada estrategia/ NEAP.

Escala de Bienestar Psicológico. Esta escala fue desarrollada en España por SánchezCánovas (2007) y validada en diferentes muestras inicialmente en mujeres de edad mediana periclimatéricas y climatéricas, pero posteriormente ampliada para su uso tanto en hombres y mujeres con edades entre los 17 y 90 años de edad. Evalúa la felicidad o satisfacción que la persona tiene en su vida a través de cuatro subescalas: Bienestar Subjetivo, Bienestar Material, Bienestar Laboral y Bienestar en Relaciones de Pareja. Está constituida por 65 ítems en una escala Likert de cinco puntos y es autoaplicable individual o colectivamente. Pueden obtenerse puntuaciones parciales por cada subescala o totales, mediante la suma algebraica de los ítems, o a través de la Escala Ponderada (Millán \& D’Aubeterre, 2011).

Cuenta con una confiabilidad que oscila entre .84 y .92 e indicadores de validez con correlaciones positivas de .89 con el Inventario de Felicidad de Oxford, de .45 con el instrumento Satisfacción con la Vida, una relación directa con la escala de Afectos Positivos (.657), e inversa con la escala de Afectos Negativos (-.471) (Sánchez-Cánovas, 2007).

La validación venezolana fue realizada por Millán y D'Aubeterre (2011), en una muestra multiocupacional, obtuvo un Alpha de Cronbach para la prueba total de .94, y entre .87 y .93 para las subescalas. Respecto a los indicadores de validez, se reportaron correlaciones positivas con Afectos Positivos (.59) y Eficacia Personal (.38), así como correlaciones negativas con Afectos Negativos (-.32), Enfermedades Físicas (-.764) y Cinismo (-.38). Es preciso anotar que en esta investigación no se administró la subescala Bienestar en las Relaciones de Pareja, debido a que no constituía un dato relevante para el tema central del estudio.

Maslach Burnout Inventory-General Survey [MBI-GS]. Se utilizó la tercera edición validada por Oramas, González y Vergara (2007) citado en Millán y D'Aubeterre (2012) a partir de la validación española de Moreno-Jiménez, Rodríquez-Carvajal y Escobar (2001) citado en Millán y D'Aubeterre (2012) la cual evalúa la presencia del síndrome de burnout para trabajadores latinoamericanos en el área de servicios. Presenta tres dimensiones: Desgaste emocional, Cinismo y Eficacia profesional.

Esta última dimensión es inversa a las anteriores, ya que refiere un carácter positivo referente a las expectativas del trabajador respecto a su profesión, sus creencias acerca de su capacidad de trabajo, su contribución a la organización, etc. Los datos obtenidos por Oramas et. al (2007), en una muestra multiocupacional de cubanos, 
El análisis abarcó descripción de la muestra, análisis de confiabilidad por consistencia interna y análisis de ítems, el cual supuso la evaluación de la capacidad discriminativa de cada ítem, de la magnitud de su varianza compartida con el resto de ítems de la prueba (comunalidad) y la agrupación de las respuestas entorno al rango establecido por la escala Likert. señalan una adecuada consistencia interna del instrumento entre .70 y .80, así como índices que corresponden a los reportados en el manual de MBI-GS de .73 y .89.

La validación en Venezuela fue realizada por Millán y D'Aubeterre (2012) a partir de la versión latinoamericana del MBI-GS, desarrollada por Oramas et. al (2007, citado por Millán y D'Aubeterre (2012). Con respecto a la confiabilidad, se observa que los valores encontrados son adecuados, aunque levemente diferentes a lo que, de acuerdo con Oramas et. al (2007) citado en Millán y D'Aubeterre, (2011), ha sido la tendencia tradicional en el estudio del MBI-GS. Concretamente, obtuvieron un Alpha de Cronbach para la prueba total de .84, de .83 en la dimensión de Cinismo, de .70 en Agotamiento emocional y de .81 en Baja eficacia profesional.

\section{Procedimiento:}

Se conformó un cuadernillo de aplicación con los instrumentos antes señalados y se incorporó adicionalmente una sección para registrar la información sociodemográfica para fines descriptivos de la muestra. También se anexó una carta de presentación explicativa del propósito del estudio y de su aporte, asegurando la confidencialidad de los datos y su uso únicamente para fines investigativos, respetando en todo momento el anonimato. Se les solicitó antes de comenzar a responder los instrumentos, su consentimiento de participación, luego de habérseles informado del propósito del estudio y del destino de los datos. Además, se les indicó también que la entrega o devolución de los instrumentos completamente llenos era una segunda evidencia expresa de su consentimiento de participación, por lo que se les invitó a destruir los cuadernillos en caso de querer abandonar el estudio. Con lo anterior se dio cumplimiento a los Artículos 57, 59, 60 del Código de Ética Profesional del Psicólogo [CEPP] (Federación de Psicólogos de Venezuela, 1981). La aplicación contó con el apoyo de estudiantes de Psicología de la Universidad Metropolitana (Venezuela), quienes contactaron a nivel personal o por medio de distintas instituciones u organizaciones a las personas que cumplían con los criterios de selección descritos anteriormente.

La información recolectada fue transcrita en Microsoft Excel 2003 y se realizó el análisis exploratorio de datos [EDA por sus siglas en inglés] y el Análisis de Consistencia Interna, el AFE con el software: Statistical Package for the Social Sciences [SPSS] versión 18, en el caso del AFC, se desarrolló con el software: Linear Structural Relations [Lisrel], versión 8.8. La migración de los datos fue automatizada con el fin de mantener la equivalencia entre las bases de datos. El análisis abarcó descripción de la muestra, análisis de confiabilidad por consistencia interna y análisis de ítems, el cual supuso la evaluación de la capacidad discriminativa de cada ítem, de la magnitud de su varianza compartida con el resto de ítems de la prueba (comunalidad) y la agrupación de las respuestas entorno al rango establecido por la escala Likert.

Para determinar el número de modelos plausibles, se llevó a cabo el AFE, cuyo método de extracción fue el de componentes principales y la rotación fue la Varimax para asegurar la cualidad de independencia estadística que debe existir en los test psicométricos. Posteriormente se llevó a cabo el AFC de los modelos, bajo la estrategia de modelos rivales (Hair et al., 1999) y utilizando el método de estimación de máxima verosimilitud basado en la matriz policórica para datos categóricas que siguen una escala tipo Likert (Hayton Allen, \& Scarpello, 2004; Hu \& Bentler, 1998; Marsh, Hau, \& Wen, 2004). Dicho método permite evaluar el nivel y el grado de ajuste de los diferentes modelos a los datos obtenidos, por medio de tres bloques o familias de indicadores, a saber, medidas de ajuste absoluto, incremental y parsimonia, 
permitiendo así determinar el modelo factorial más verosímil a aquél que posea todos los indicadores de ajuste absoluto y que acumule, a su vez, el mayor número de indicadores de ajuste incremental y parsimonia de los modelos rivales evaluados.

\section{Resultados y Discusión}

La muestra válida quedó conformada por 1681 trabajadores activos, teniendo presente la verificación de la calidad del tamaño de la muestra obtenida por los criterios de representatividad estadística, potencia (Hair et al., 1999) y autoridad (Prieto \& Muñiz, 2000). La mayoría de los participantes fueron del Distrito Capital de Venezuela $(82,87 \%)$ y el resto de diversas ciudades del país como Guarenas-Guatire (8\%) y Altos Mirandinos (2\%), entre otras, y cuya población para el año 2014, según datos oficiales del Instituto Nacional de Estadística [INE] de Venezuela, fue de 375.294 trabajadores (INE, 2012).

En términos descriptivos, la edad de la muestra osciló entre 16 y 73 años, con un promedio de 37.51, (Mediana de 36 años), con una leve asimetría positiva (As=0.36) lo cual indica que los datos se distribuyen cercanos a la media, siendo una distribución platicúrtica $(\mathrm{Cu}=-0,70)$. Sus ciclos vitales (Papalia \& Olds, 1998), se agrupan en: $3 \%$ adolescentes, $59 \%$ adultos tempranos, $37 \%$ adultos intermedios y el $2 \%$ eran adultos tardíos.

En cuanto al nivel educativo, el 1.13\% culminó educación básica o primaria, el 17.01\% era bachiller, el $22.61 \%$ era técnico superior, el $36.17 \%$ universitario y, finalmente, aquellos con algún estudio de postgrado se dividieron en: $13.44 \%$ especialista, $7.38 \%$ magíster y $0,95 \%$ doctor (el $1.24 \%$ no reportó).

En relación con el estado civil, $41.17 \%$ eran solteros, $7.73 \%$ estaban en unión libre, $40.99 \%$ casados, $8.21 \%$ divorciados, $0.65 \%$ viudo(a)s y $1.08 \%$ no aportó información.

Respecto al tipo de contratación, el 32.84\% de los trabajadores laboraba a tiempo determinado, el $58.95 \%$ a tiempo indeterminado, el $0.06 \%$ era empleado de libre nombramiento y remoción y el $8.15 \%$ no contestó esta información.

En referencia al nivel socioeconómico medido con el sistema Graffar para Venezuela (Seijas, 2003; Millán \& D'Aubeterre, 2012), el 23.20\% pertenece a la clase obrera, $1,55 \%$ marginal, $34.15 \%$ a la clase media-baja, $30.34 \%$ a la clase media y $10.77 \%$ a la clase alta, (1.08\% no reportó).

Finalmente, respecto a los años de antigüedad en el trabajo, la distribución osciló entre 0 y 50 años de antigüedad, un promedio de 6.92 años, un \% de variabilidad y una asimetría marcadamente positiva (As $=1.77)$.

Con respecto al análisis de ítems, se puede observar en las tablas 1 y $\underline{2}$ que, en cuanto al rango, las respuestas abarcan la totalidad de las escalas. Por lo tanto, una vez seleccionadas todas las opciones de respuesta, se puede considerar la amplitud de cinco puntos como pertinente para ambos instrumentos. Se aprecia también que todos los ítems presentan una capacidad discriminativa mayor a .30, lo que supone que son útiles para discriminar entre aquellos que se perciben como acosados y los que no. Asimismo, todos los ítems presentan medias bajas con respecto al recorrido de la escala de cinco puntos, indicando que aun cuando las respuestas abarcan todo el rango esperado, se concentran en la puntuación más baja. 
También puede observarse en la tabla 1 que la eliminación del ítem 23 del NAQ-R no afecta considerablemente el coeficiente Alpha de Cronbach del instrumento, por lo que dicho ítem no implica un aporte a la confiabilidad total.

Adicionalmente, solo el coeficiente de variación media [C. $V_{\text {media }}$ ] del ítem 22, es menor al 30\%, lo que implica homogeneidad en el patrón de respuestas a dichos ítems.

Por otra parte, la eliminación del ítem 1, 2 y 58 el LIPT-60 no afecta considerablemente el coeficiente Alpha de Cronbach del instrumento, por lo que dichos ítems no implican un aporte a la confiabilidad total y ningún coeficiente de variación media (C.V media) es menor al 30\%, lo que implica homogeneidad en el patrón de respuestas a dichos ítems.

Tabla 1. Análisis de ítems NAQ-R

\begin{tabular}{|c|c|c|c|c|c|}
\hline Ítem & Promedio & $\begin{array}{l}\text { Desviación } \\
\text { Estándar }\end{array}$ & $\begin{array}{c}\text { Capacidad } \\
\text { discriminativa }\end{array}$ & $\begin{array}{l}\text { Alfa de Cronbach si } \\
\text { se elimina el item }\end{array}$ & Comunalidad \\
\hline $\begin{array}{l}\text { p1 } \\
\text { p2 } \\
\text { p3 } \\
\text { p4 } \\
\text { p5 } \\
\text { p6 } \\
\text { p7 } \\
\text { p8 } \\
\text { p9 } \\
\text { p10 } \\
\text { p11 } \\
\text { p12 } \\
\text { p13 } \\
\text { p14 } \\
\text { p15 } \\
\text { p16 } \\
\text { p17 } \\
\text { p18 } \\
\text { p19 } \\
\text { p20 } \\
\text { p21 } \\
\text { p22 } \\
\text { p23 }\end{array}$ & $\begin{array}{l}1,27 \\
1,13 \\
1,29 \\
1,22 \\
1,29 \\
1,19 \\
1,16 \\
1,27 \\
1,09 \\
1,18 \\
1,26 \\
1,16 \\
1,26 \\
11,32 \\
1,20 \\
1,13 \\
1,14 \\
1,36 \\
1,20 \\
1,18 \\
1,39 \\
1,05 \\
1,06\end{array}$ & $\begin{array}{l}.555 \\
.451 \\
.627 \\
.583 \\
.617 \\
.533 \\
.492 \\
.603 \\
.362 \\
.526 \\
.632 \\
.473 \\
.646 \\
.659 \\
.530 \\
.483 \\
.425 \\
.821 \\
.577 \\
.535 \\
.826 \\
.315 \\
.333\end{array}$ & $\begin{array}{l}.591 \\
.668 \\
.612 \\
.672 \\
.665 \\
.675 \\
.685 \\
.706 \\
.569 \\
.688 \\
.725 \\
.712 \\
.773 \\
.754 \\
.668 \\
.647 \\
.672 \\
.624 \\
.690 \\
.651 \\
.645 \\
.484 \\
.334\end{array}$ & 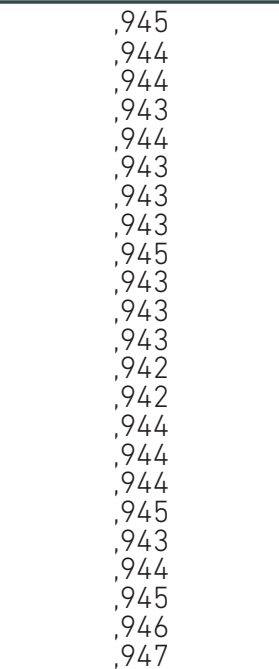 & 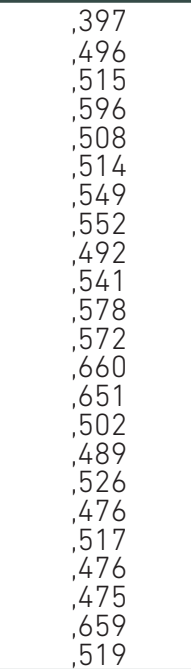 \\
\hline
\end{tabular}

Con respecto a la consistencia interna total de cada instrumento, en la tabla 3 , se observa que el valor del coeficiente Alpha de Cronbach fue de .95 para el NAQ-R y de 98 para el LIPT-60 interpretándose como "Excelente", para ambos instrumentos, según el criterio de Prieto y Muñiz (2000).

Se determinó que era factible considerar la presencia de alguna estructura factorial subyacente a los datos en ambos instrumentos (Pardo \& Ruiz, 2002; Hair et al., 1999) puesto que se cumplieron con los supuestos estadísticos referidos, a saber, el determinante obtenido fue igual al criterio de $0(\mathrm{D}=.000)$, la medida de adecuación muestral de $\mathrm{KMO}$ fue mayor al criterio de 0.5 ( $\mathrm{KMO}=.960$ para el $\mathrm{NAQ}-\mathrm{R}$ y $\mathrm{KMO}=.981$ para el LIPT-60) y la medida de significancia de la prueba de esfericidad de Bartlett fue menor al criterio de 0.05 ( $\left.p_{\text {Bartlett }}=0.000\right)$.

Como puede apreciarse en las tablas $\underline{4}$ y $\underline{5}$, se describen las estructuras factoriales obtenidas para cada instrumento en investigaciones previas, identificándose la presencia de seis estructuras factoriales distintas para el NAQ-R y de 7 para LIPT-60, tanto de primer como de segundo nivel. Esta diversidad es consecuencia del sesgo de indeterminación factorial del AFE. En este último aspecto se observa que en el criterio de autovalor acumulado superior a 1 y el criterio de porcentaje de la varianza 
Tabla 2. Análisis de ítems LIPT-60

\begin{tabular}{|c|c|c|c|c|c|}
\hline Ítem & Promedio & $\begin{array}{l}\text { Desviación } \\
\text { Estándar }\end{array}$ & $\begin{array}{c}\text { Capacidad } \\
\text { discriminativa }\end{array}$ & $\begin{array}{l}\text { Alfa de Cronbach si } \\
\text { se elimina el item }\end{array}$ & Comunalidad \\
\hline $\begin{array}{l}\text { p1 } \\
\text { p2 } \\
\text { p3 } \\
\text { p4 } \\
\text { p5 } \\
\text { p6 } \\
\text { p7 } \\
\text { p8 } \\
\text { p9 } \\
\text { p10 } \\
\text { p11 } \\
\text { p12 } \\
\text { p13 } \\
\text { p14 } \\
\text { p15 } \\
\text { p16 } \\
\text { p17 } \\
\text { p18 } \\
\text { p19 } \\
\text { p20 } \\
\text { p21 } \\
\text { p22 } \\
\text { p23 } \\
\text { p24 } \\
\text { p25 } \\
\text { p26 } \\
\text { p27 } \\
\text { p28 } \\
\text { p29 } \\
\text { p30 } \\
\text { p31 } 312 \\
\text { p32 } \\
\text { p33 } \\
\text { p34 } \\
\text { p35 } \\
\text { p36 } \\
\text { p37 } \\
\text { p38 } \\
\text { p39 } \\
\text { p40 } \\
\text { p41 } \\
\text { p42 } \\
\text { p43 } \\
\text { p44 } \\
\text { p45 } \\
\text { p46 } \\
\text { p47 } \\
\text { p48 } \\
\text { p49 } \\
\text { p50 } \\
\text { p51 } \\
\text { p52 } \\
\text { p53 } \\
\text { p54 } \\
\text { p55 } \\
\text { p56 } \\
\text { p57 } \\
\text { p58 } \\
\text { p59 } \\
\text { p60 }\end{array}$ & 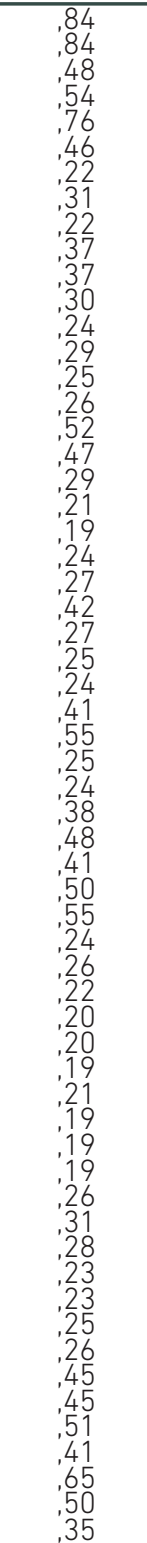 & 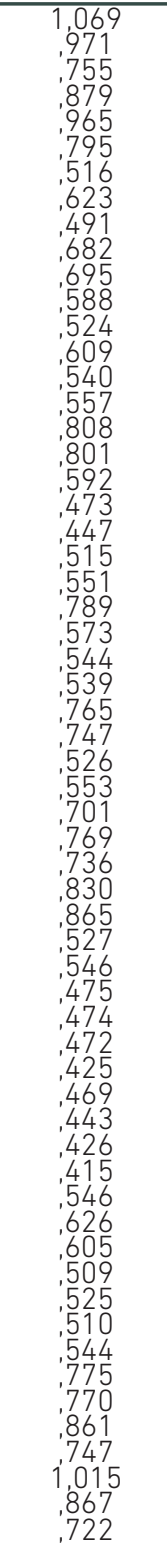 & 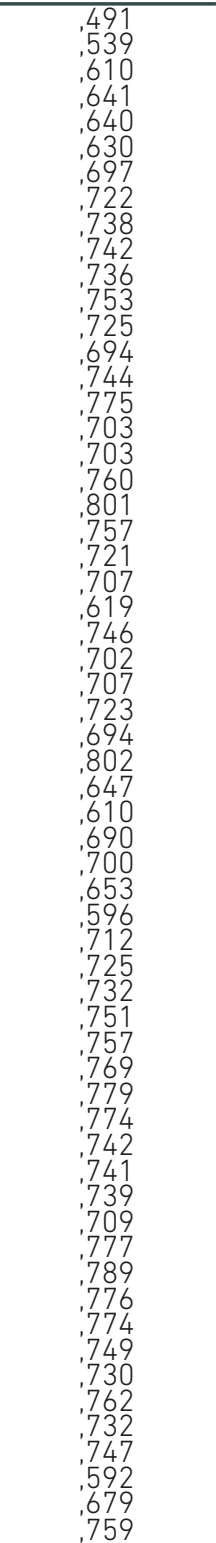 & $\begin{array}{l}.982 \\
.982 \\
.981 \\
.981 \\
.981 \\
.981 \\
.981 \\
.981 \\
.981 \\
.981 \\
.981 \\
.981 \\
.981 \\
.981 \\
.981 \\
.981 \\
.981 \\
.981 \\
.981 \\
.981 \\
.981 \\
.981 \\
.981 \\
.981 \\
.981 \\
.981 \\
.981 \\
.981 \\
.981 \\
.981 \\
.981 \\
.981 \\
.981 \\
.981 \\
.981 \\
.981 \\
.981 \\
.981\end{array}$ & 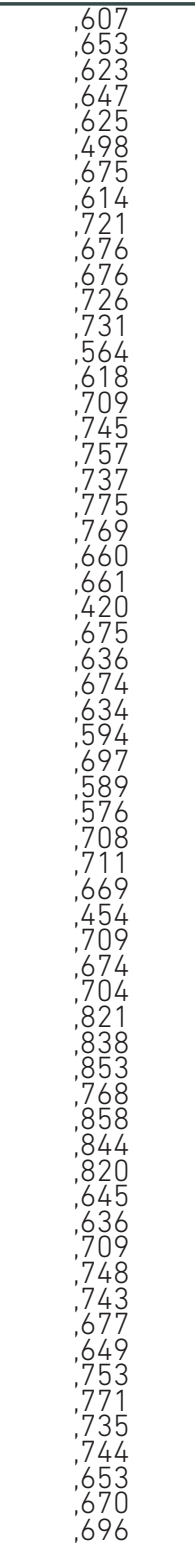 \\
\hline
\end{tabular}

Tabla 3. Indicadores de consistencia interna (Alfa de Cronbach)

\begin{tabular}{cccccccc}
\hline Instrumento & $\begin{array}{c}\text { Alfa de } \\
\text { Cronbach }\end{array}$ & $\begin{array}{c}\boldsymbol{N} \text { de } \\
\text { ítems }\end{array}$ & Dimensión 1 & Dimensión 2 & Dimensión 3 & Dimensión 4 & Dimensión 5 \\
\hline NAQ-R &, 946 & 23 &, 899 &, 853 &, 864 &, 637 & \\
LIPT-60 &, 981 & 60 &, 972 &, 972 &, 890 &, 836 &, 740 \\
\hline
\end{tabular}

acumulada superior al 60\%, coinciden con la presencia de modelos de dos factores (Figura 1) y tres factores (Figura 2), respectivamente. Sin embargo, en la tabla 4 se muestra que el 6to factor es residual porque ninguno de los ítems posee su mayor carga factorial en él. Por ello, se concluyó que uno de los modelos posibles es el de cinco factores (Tabla 4), el cual acumuló un $56.77 \%$ de la varianza total y coincidió con el criterio a priori del modelo original de Blanch, Sahagún y Cervantes (2010). 
Tabla 4. Modelos obtenidos del NAQ

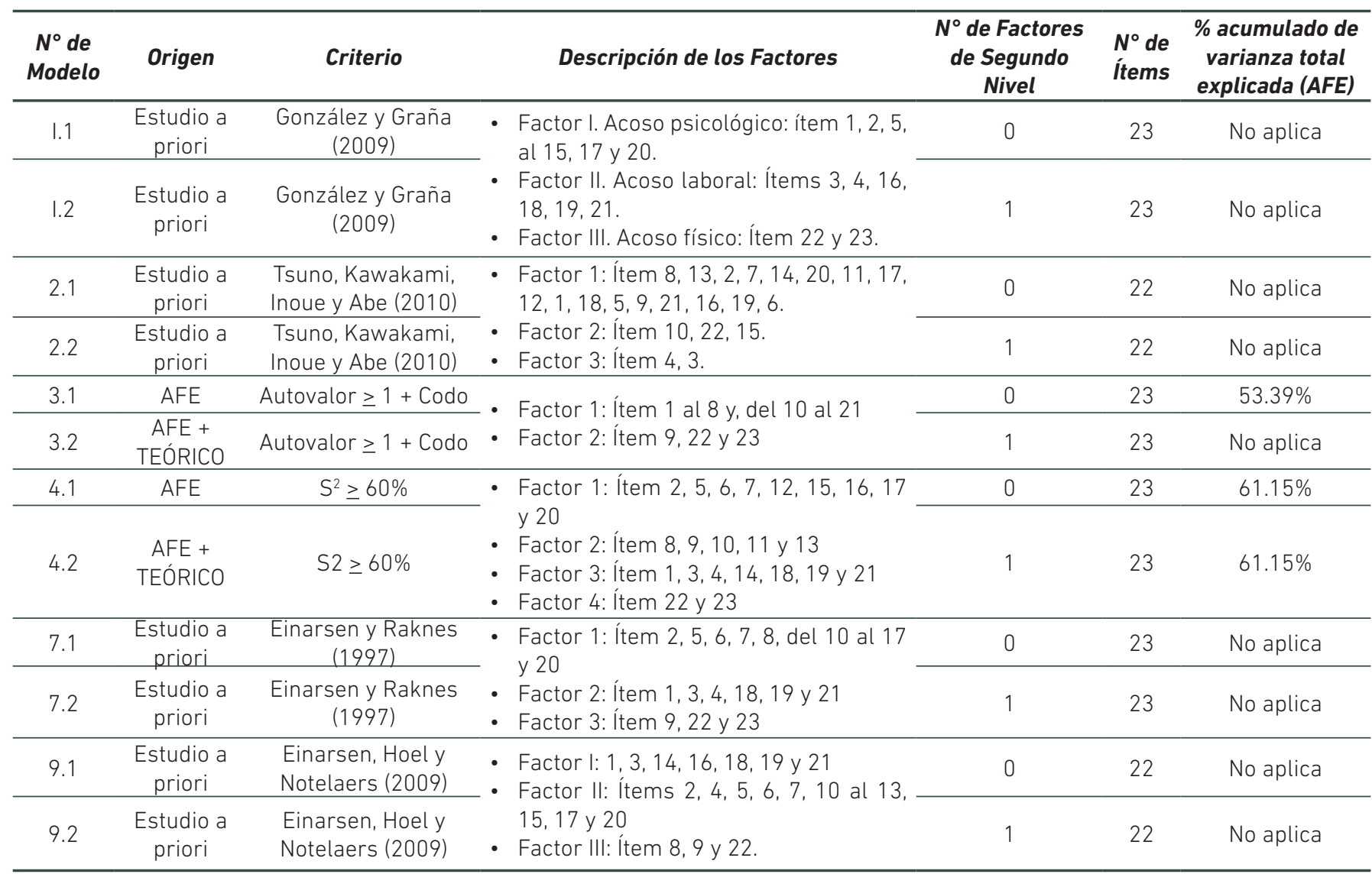

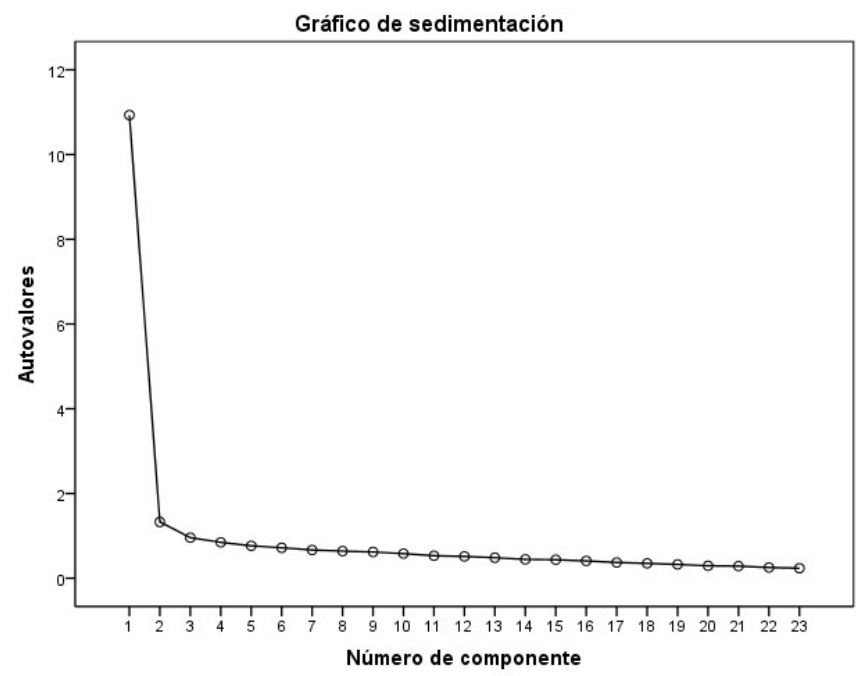

Q

Figura 1. Gráfico de sedimentación AFE del NAQ-R

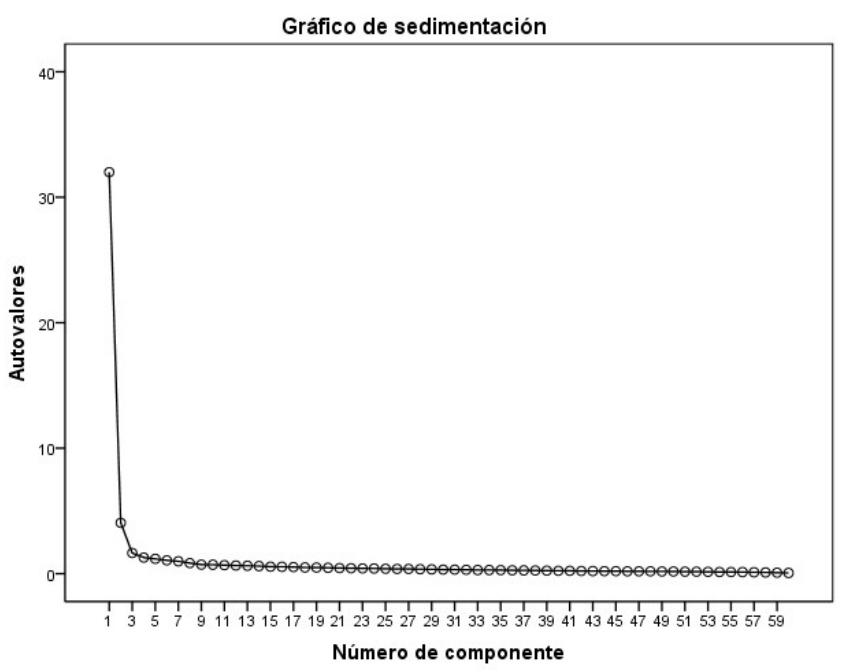

Q

Figura 2. Gráfico de sedimentación AFE del LIPT-60 
Tabla 5. Modelos obtenidos del LIPT

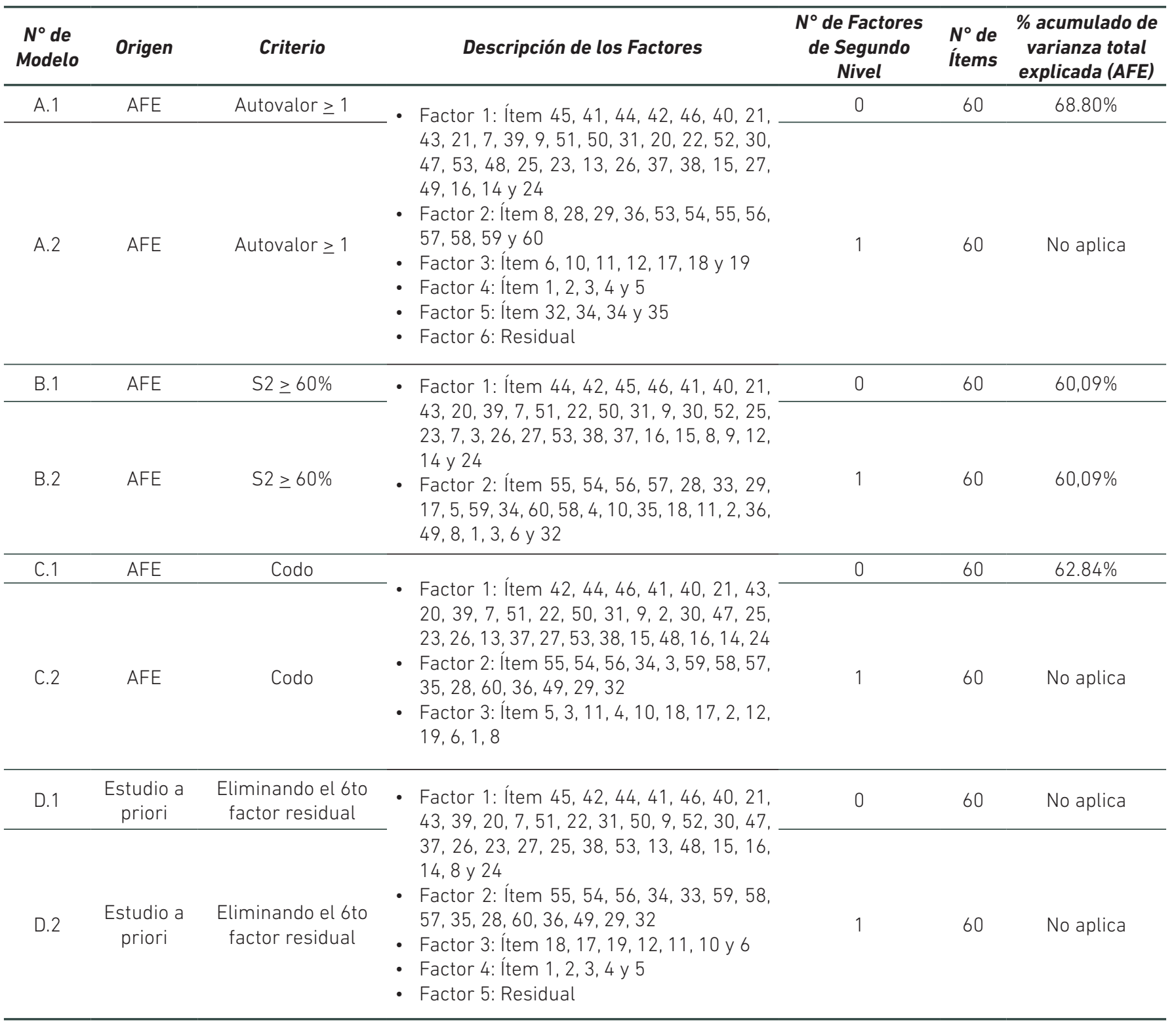

Como solución al sesgo de indeterminación factorial del AFE, está la comparación de los indicadores de ajuste de cada modelo, calculado a partir del AFC. Así, en las tablas $\underline{6}$ y $\underline{7}$ se encuentran tanto los indicadores de ajuste absoluto como los incremental y de parsimonia, de los cuales puede concluirse que los más verosímiles son los modelos H.1 y el 4.1, dado que son los que mantienen un mayor número de indicadores de ajuste dentro del límite considerado como aceptable. Específicamente, en ambos el valor de su $\chi^{2}$ es el más bajo, así como también la razón $\chi^{2} /$ gl, que es un indicador que se utiliza para corregir la hipersensibilidad del $\chi^{2}$ a muestras grandes. Sin embargo, Hair et al. (1999) señalan que, en casos como el presente estudio, en el que la muestra supera a las 200 observaciones, estos indicadores se hacen hipersensibles por lo que: se anima al investigador a completar ésta medida con otras medidas de ajuste en todos los casos, inclusive con la razón $\chi^{2} / \mathrm{gl}$, que se utilizaría mejor para hacer comparaciones entre modelos alternativos eligiendo entre estos al menor. 
Por ser el más alto, el valor del Índice de Bondad de Ajuste [GFI] es el mejor ajuste de todos los modelos considerados. Por otro lado, el valor del Error de aproximación cuadrático medio [RMSEA], que es el indicador más importante para el análisis de modelos confirmatorios bajo la estrategia de modelos rivales (Hair et al., 1999), no confirmó cuál de los modelos es el más adecuado porque los valores observados para los modelos H.1 y el 4.1 están fuera de los límites de 0.05 y 0.08 puntos. No obstante, Hair et al. (1999), también señalan que cuando la muestra es mayor a 100 casos, se puede interpretar éste valor como dentro del "límite de umbral superior" (p.660) cuando se encuentra por debajo de 0.10 puntos, tal como sucede en este caso.

Con respecto a los indicadores de ajuste incremental, solo el modelo H.1, cumple con el valor del límite mínimo de 0.90 puntos, a excepción del Índice de Bondad de Ajuste Ajustado [AGFI] que, en ambos modelos, se encontró levemente por encima de dicho valor. En este particular, y considerando nuevamente el gran tamaño de la muestra, el criterio de evaluación se cambia a 0.10 , interpretando entonces estos valores como "dentro del límite marginal aceptable" (Hair et al., 1999, p.659).

De hecho, en lo que respecta a los indicadores de ajuste incremental Hair et al. (1999), señalan que:

Aunque el umbral de 0.90 no tiene fundamento estadístico, la experiencia y la investigación práctica han demostrado su utilidad en la distinción entre modelos aceptables y no aceptables. Sin embargo, todas las medidas del ajuste incremental exceden de 0.80 y el verdadero contraste proviene de la comparación del modelo propuesto frente a los modelos alternativos (p. 662).

No se obtuvieron diferencias sustanciales en los valores de los indicadores de ajuste de parsimonia de dichos modelos, ya que la diferencia entre los valores de cada uno de ellos no se encontró dentro del límite de los 0.06 a 0.09 puntos (Hair et al., 1999, p. 684). Se determina entonces que el modelo H.1. es el más verosímil ya que es el que posee una mayor cantidad de indicadores.

Tabla 6. Indicadores de ajuste AFC NAQ-R

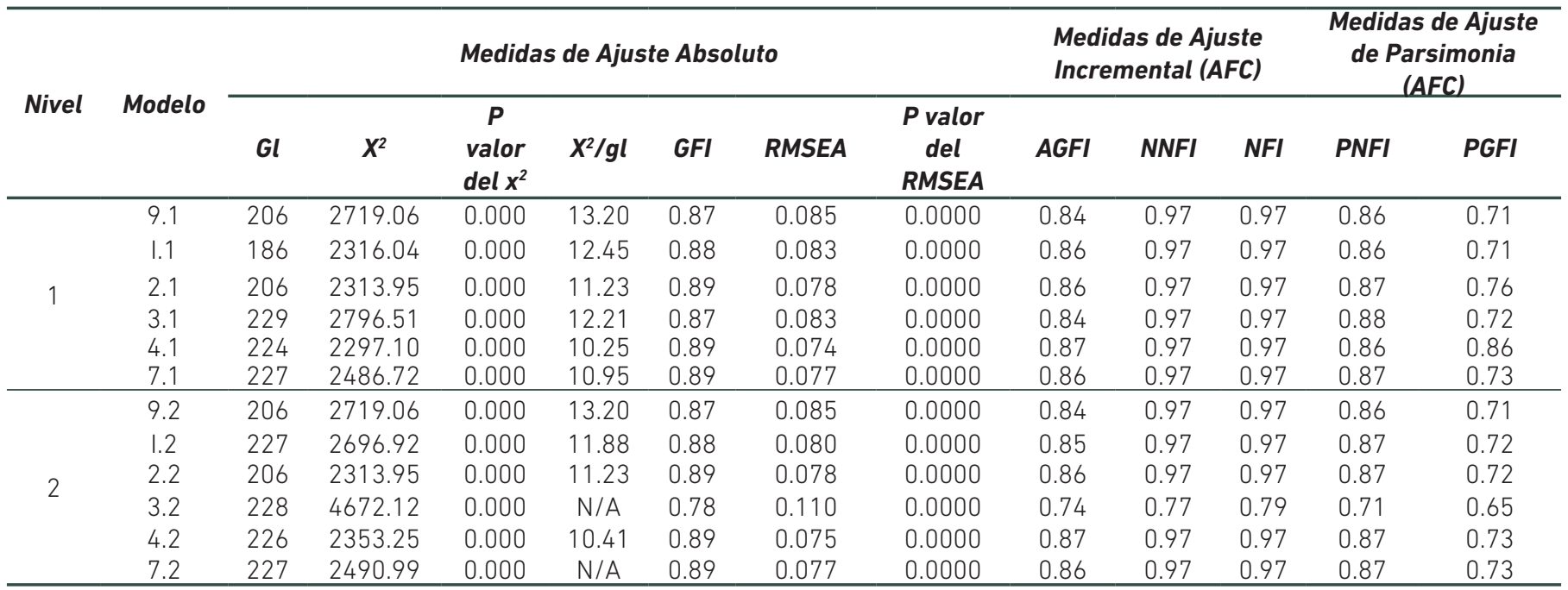


Pág 56

Tabla 7. Indicadores de ajuste AFC LIPT

\begin{tabular}{|c|c|c|c|c|c|c|c|c|c|c|c|c|c|}
\hline \multirow{2}{*}{ Nivel } & \multirow{2}{*}{ Modelo } & \multicolumn{7}{|c|}{ Medidas de Ajuste Absoluto } & \multicolumn{3}{|c|}{$\begin{array}{l}\text { Medidas de Ajuste } \\
\text { Incremental (AFC) }\end{array}$} & \multicolumn{2}{|c|}{$\begin{array}{c}\text { Medidas de Ajuste } \\
\text { de Parsimonia } \\
\text { (AFC) }\end{array}$} \\
\hline & & Gl & $X^{2}$ & $\begin{array}{c}P \\
\text { valor } \\
\text { del } x^{2} \\
\end{array}$ & $X^{2} / g l$ & GFI & RMSEA & $\begin{array}{c}\text { P valor } \\
\text { del } \\
\text { RMSEA }\end{array}$ & AGFI & NNFI & NFI & PNFI & PGFI \\
\hline \multirow[t]{7}{*}{1} & A. 1 & 1700 & 26124.26 & 0.000 & 15.37 & 0.66 & 0.092 & 0.0000 & 0.63 & 0.98 & 0.98 & 0.94 & 0.61 \\
\hline & B.1 & 1709 & 34113.07 & 0.000 & 19.96 & 0.60 & 0.110 & 0.0000 & 0.57 & 0.97 & 0.97 & 0.94 & 0.56 \\
\hline & C.1 & 1707 & 27150.35 & 0.000 & 15.91 & 0.65 & 0.094 & 0.0000 & 0.62 & 0.98 & 0.97 & 0.94 & 0.61 \\
\hline & D.1 & 1646 & 25952.58 & 0.000 & 15.77 & 0.66 & 0.094 & 0.0000 & 0.63 & 0.98 & 0.97 & 0.94 & 0.61 \\
\hline & F.1 & 1160 & 21476.10 & 0.000 & 18.51 & 0.66 & 0.100 & 0.0000 & 0.63 & 0.97 & 0.97 & 0.92 & 0.60 \\
\hline & G.1. & 1704 & 25751.01 & 0.000 & 15.11 & 0.66 & 0.092 & 0.0000 & 0.64 & 0.98 & 0.98 & 0.94 & 0.62 \\
\hline & H.1 & 935 & 14641.72 & 0.000 & 15.66 & 0.72 & 0.093 & 0.0000 & 0.69 & 0.97 & 0.97 & 0.92 & 0.97 \\
\hline \multirow[t]{7}{*}{2} & A. 2 & 1647 & 25626.02 & 0.000 & 15,56 & 0,66 & 0,093 & 0.0000 & 0,63 & 0,98 & 0,98 & 0,94 & 0,61 \\
\hline & B.2 & 1707 & 32062,98 & 0.000 & $\mathrm{~N} / \mathrm{A}$ & 0,60 & 0,100 & 0.0000 & 0,57 & 0,95 & 0,95 & 0,92 & 0,56 \\
\hline & C. 2 & 1648 & 29031,58 & 0.000 & 17,62 & 0,63 & 0,099 & 0.0000 & 0,60 & 0,97 & 0,97 & 0,94 & 0,59 \\
\hline & D.2 & 1706 & 26483,63 & 0.000 & 15,52 & 0,66 & 0,093 & 0.0000 & 0,63 & 0,98 & 0,97 & 0,94 & 0,61 \\
\hline & F.2 & 1163 & 25149,62 & 0.000 & 21,51 & 0,63 & 0,110 & 0.000 & 0,59 & 0,97 & 0,97 & 0,92 & 0,57 \\
\hline & G.2 & 1706 & 25953,85 & 0.000 & 15,21 & 0,66 & 0,092 & 0.000 & 0,64 & 0,98 & 0,98 & 0,94 & 0,62 \\
\hline & H.2 & 940 & 15479,87 & 0.000 & 16,47 & 0,71 & 0,096 & 0.000 & 0,68 & 0,97 & 0,97 & 0,92 & 0,64 \\
\hline
\end{tabular}

En relación con la confiabilidad por consistencia interna para cada una de estas estructuras, para el modelo H.1. [LIPT] fue de .98 y para el modelo 4.1 [NAQR] fue de 0.94 , siendo consideradas ambas como excelentes según criterios definidos por Prieto y Muñiz (2000).

En relación a la naturaleza (ponderada o sumada) del cálculo del puntaje total de cada sujeto en cada uno de los cinco factores del modelo confirmado H.1, se recomienda el uso de la estrategia ponderada, ya que asegura el supuesto de independencia entre los factores del modelo debido a que confirma que la correlación entre factores es igual a 0 . Este es un supuesto de la teoría clásica de los test, en su búsqueda de medidas sin correlación entre los errores de medida y sin correlación entre la estimación del puntaje verdadero que hace el test y su error de medida (Muñiz, 1998). Por esta razón, deberían entonces utilizarse los coeficientes betas [ $\beta$ ], una vez se haya estandarizado las respuestas de los sujetos en cada ítem de acuerdo con la media y desviación de este grupo, que fungiría como normativo.

Finalmente, para el cálculo de la validez predictiva de ambos instrumentos, se tenían dos aproximaciones de cálculo, considerando que tanto el bienestar y el burnout puede operacionalizarse como una variable de nivel cuantitativo y escala de intervalo (puntaje percentilar) o de nivel cualitativo de escala ordinal (a partir de los puntos de cortes establecidos en las normas descritas por Millán y D'Aubeterre (2011; 2012). En este orden de ideas, la muestra estuvo compuesta por un $7 \%$ de participantes con bienestar psicológico muy bajo, 12\% con nivel bajo, 25\% con nivel medio, $17 \%$ con un nivel alto y, finalmente, $39 \%$ con un nivel muy alto. Por otra parte, con el indicador de burnout, se obtuvo una muestra compuesta por un $15 \%$ con nivel muy bajo, $20 \%$ con un nivel bajo, 30\% con un nivel medio, $20 \%$ con un nivel alto y, por último, $15 \%$ con un nivel alto.

Por tal motivo, se estimaron diferentes coeficientes de correlación multivariante siguiendo la recomendación de Furham (1995), quien señala que una de las limitaciones en los estudios relacionados con características personales en el ámbito organi- 
zacional es el uso de "correlaciones simples en lugar de correlaciones parciales o más bien preferiblemente, estadísticos multivariantes para prevenir los errores tipo "I (encontrar más diferencias significativas de las reales)" (p. 35). Es así como, en la tabla 8 , se observa el uso del análisis discriminante y la regresión logística cuando se operacionalizó el bienestar psicológico y el burnout como una variable de nivel cualitativo y de escala ordinal y la regresión múltiple, cuando se operacionalizó como una variable de nivel cuantitativo y de escala intervalo. Como predictores se utilizaron los puntajes factoriales del modelo 4.1 [NAQR] y del modelo H.1 [LIPT].

En la tabla 8, al comparar la correlación más alta del modelo 4.1 con la del H.1 en burnout, se observa que el LIPT es el instrumento de acoso que mejor la predice $\left(R_{\text {modelo }} 4.1=0.33 ; R_{\text {modelo }} H .1=0.50\right)$. En el caso del bienestar, se observa nuevamente que el LIPT es el que mejor lo predice en comparación con el NAQR $\left(R_{\text {modelo }} 4.1=\right.$ $0.24 ; R_{\text {modelo }} H .1=0.35$ ), con lo cual se justifica la validez empírica del mismo, más aún cuando predice mejor el nivel de burnout alto.

Tabla 8. Correlación multivariante entre el NAQ-R y el LIPT con bienestar psicológico y burnout

\begin{tabular}{|c|c|c|c|c|c|c|c|}
\hline \multirow{2}{*}{ Test } & \multirow{2}{*}{ Función } & \multicolumn{2}{|c|}{$\begin{array}{l}\text { Coeficiente de } \\
\text { Correlación }\end{array}$} & \multicolumn{2}{|c|}{ P-Valor } & \multicolumn{2}{|c|}{ \% de Correcta Predicción } \\
\hline & & Bienestar & Burnout & Bienestar & Burnout & Bienestar & Burnout \\
\hline \multirow{3}{*}{$\begin{array}{l}\text { NAQR } \\
(4.1)\end{array}$} & Discriminante & 0,21 & 0,25 & 0,000 & 0,000 & $48,60 \%$ & $59,10 \%$ \\
\hline & Logística & 0,22 & 0,26 & 0,428 & 0,004 & $48,80 \%$ & $59,20 \%$ \\
\hline & $\begin{array}{l}\text { Regresión } \\
\text { Múltiple }\end{array}$ & 0,24 & 0,33 & 0,000 & 0,000 & No Aplica & No Aplica \\
\hline \multirow{3}{*}{$\begin{array}{l}\text { LIPT } \\
(H .1)\end{array}$} & Discriminante & 0,31 & 0,39 & 0,000 & 0,000 & $53,30 \%$ & $62,10 \%$ \\
\hline & Logística & 0,33 & 0,41 & 0,000 & 0,000 & $53,10 \%$ & $62,90 \%$ \\
\hline & $\begin{array}{l}\text { Regresión } \\
\text { Múltiple }\end{array}$ & 0,35 & 0,50 & 0,000 & 0,000 & No Aplica & No Aplica \\
\hline
\end{tabular}

Estos resultados tienen varias implicaciones para ayudar a solventar lo que Kahale (2015) señaló como dificultad a nivel legal al momento de "seleccionar la técnica reguladora más idónea para la tutela judicial efectiva del derecho del lesionado, así como la fijación de las consecuencias jurídicas derivadas de la conducta ilícita" (p. 19). En este sentido, es necesario considerar que la validez predictiva "se refiere al uso de un instrumento para estimar alguna conducta criterio que es externa al mismo instrumento de medición (Nunally \& Bernstein, 1995, p. 104), y que, por consiguiente, en este caso es factible considerar al burnout como criterio externo al mobbing, aun cuando éste sea un constructo psicológico, ya que adquiere independencia teórica por la vía legal al ser tipificado como una enfermedad ocupacional (NT-02-2008) e incluso al caracterizar una serie de consecuencias objetivas para quien lo ejecute (INSPASEL, 2013). Esta lógica es la misma que mantienen los métodos de evaluación SLM (León-Pérez et al., 2004) cuando logran predecir de forma adecuada, como sucede con el LIPT-45, los indicadores de salud de los trabajadores, tal como sucedió con los estudios de León-Pérez, et al. (2004), Mayhew y Chappell

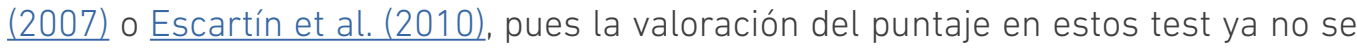
hace de forma arbitraria sino que se guía por la variación concomitante que poseen con indicadores de salud psicológica en el trabajo. En tal virtud, la interpretación del nivel de acoso en el LIPT-45 vendría dada por las siguientes ecuaciones logísticas, que reproducen la predicción de un nivel alto de burnout y de un nivel bajo de bienestar psicológico (Tablas $\underline{9}$ y $\underline{10}$ ) con punto de corte 0.50 para cada uno de ellos. 
Tabla 9. Coeficientes $\beta_{\text {LIPT-45 }}$ para la predicción del bienestar psicológico

\begin{tabular}{lcccccc}
\hline \multicolumn{1}{c}{ Factores componentes del LIPT-45 } & B & $\begin{array}{c}\text { Error } \\
\text { estándar }\end{array}$ & Wald & gl & Sig. & Exp (B) \\
\hline FAC1 (Comprometer su salud) &,- 132 &, 063 & 4,431 & 1 &, 035 &, 877 \\
FAC2 (Limitar su contacto social) &,- 140 &, 065 & 4,630 & 1 &, 031 &, 869 \\
FAC3 (Desacreditar su capacidad profesional y laboral) &,- 336 &, 062 & 29,472 & 1 &, 000 &, 715 \\
FAC4 (Limitar su comunicación) &,- 484 &, 068 & 51,306 & 1 &, 000 &, 617 \\
FAC5 (Desprestigiarlo ante sus compañeros) &,- 248 &, 060 & 17,035 & 1 &, 000 &, 781 \\
Constante & 2,109 &, 083 & 642,273 & 1 &, 000 & 8,237 \\
\hline
\end{tabular}

Tabla 10. Coeficientes $\beta_{\text {LPT }-45}$ para la predicción de burnout

\begin{tabular}{|c|c|c|c|c|c|c|}
\hline Factores componentes del LIPT-45 & B & $\begin{array}{c}\text { Error } \\
\text { estándar }\end{array}$ & Wald & gl & Sig. & $\operatorname{Exp}(B)$ \\
\hline FAC1 (Comprometer su salud) &,- 326 & .057 & 33,088 & 1 &, 000 &, 722 \\
\hline FAC2 (Limitar su contacto social) &, 229 & 059 & 14,876 & 1 & .000 & 1,257 \\
\hline FAC3 (Desacreditar su capacidad profesional y laboral) &, 552 &, 067 & 67,254 & 1 &, 000 & 1,737 \\
\hline FAC4 (Limitar su comunicación) & .439 &, 055 & 63,203 & 1 &, 000 & 1,551 \\
\hline FAC5 (Desprestigiarlo ante sus compañeros) &, 108 &, 063 & 2,901 & 1 &, 089 & 1,114 \\
\hline Constante &,- 323 & 053 & 37,293 & 1 &, 000 &, 724 \\
\hline
\end{tabular}

Igualmente, tampoco se podría aludir como limitante al uso del LIPT-45, y a esta norma de corrección para Venezuela en tanto predictor de un burnout alto y un bajo bienestar, a la naturaleza subjetiva o teórica de esta medida (y en menoscabo de ésta por indicadores más objetivos como por ejemplo conductas de acoso), ya que, por un lado, a nivel legal se garantiza la independencia de lo mental (que es por definición de naturaleza subjetiva), de lo físico y lo social (WHO, 1946; LOS, 1998), además de que estas definiciones de salud, tanto a nivel internacional como en la legislación venezolana, aseguran que lo mental no se encuentra subordinado a lo físico. Por otro lado, como señalan Anastasi y Urbina (1998), "la aplicación, calificación e interpretación de los resultados [de un test psicológico] serán objetivas en la medida en que sean independientes al juicio subjetivo del investigador (p. 7), lo cual se logra con las normas de corrección aquí presentada tanto en la puntuación de los factores componentes del acoso psicológico en el LIPT-45 (desprestigio personal, desprestigio laboral, intimidación encubierta e intimidación manifiesta), tanto en la estimación a partir de estos factores de los niveles altos de burnout y bajos de bienestar psicológico.

De la misma manera, al observar la calidad predictiva del LIPT-45 y la direccionalidad de sus factores componentes, se confirma que se cumple la direccionalidad esperada teóricamente (a excepción del factor de comprometer su salud, que es inverso al burnout, aunque mantiene una relación inversa con bienestar psicológico, explicándose quizás esta diferencia en términos del efecto sobre la salud - enfermedad o la mayor cronicidad del burnout como afección psicosocial del trabajo). De hecho, a mayor puntaje en cada uno de los factores componentes del LIPT-45, mayor probabilidad de tener un bienestar psicológico bajo y un burnout alto, por lo que no sólo hay una consistencia teórica sino una predictibilidad de los indicadores de salud psicológica de los trabajadores que coincide con lo señalado por Nunally y Bernstein (1995): "ninguna teoría aparentemente sana puede sustituir la falta de correlación entre el predictor y el criterio" (p. 106). Ahora bien, aunque existe capacidad predictiva, que es un principio básico de la ciencia (Bunge, 1966) 
Pág 59

La misma OIT reconoce la importancia que tiene la intervención secundaria para inmunizar al individuo frente a la enfermedad, comprendiendo todas aquellas intervenciones de carácter formativa (Hurrel et al., p. 5.5), lo cual se facilitaría aún más si se posee una medición regular y científicamente validada que informe la prevalencia del mobbing y su correlato sobre la salud psicológica de los trabajadores. y un aspecto clave en la posibilidad de explicar la salud (Gómez-Restrepo \& Delgado, 2006), también es cierto que el hecho de que exista correlación no implica necesaria y suficientemente la posibilidad de explicación de la enfermedad o de la salud, pues existen otros factores como la secuencia temporal de los eventos, la relación dosis-respuesta, la plausibilidad de la asociación observada, la evidencia experimental y la reversibilidad, que no son abordados desde la evidencia de correlación. Este aspecto de la causalidad es de vital importancia en el contexto legal para asegurar responsabilidad del patrono al momento de fallar a favor del demandante en los casos judiciales de demanda por acoso psicológico en el trabajo, tal como se observó a nivel internacional cuando en el año 2009 la OIT retiró al mobbing de la listas de enfermedades laborales hasta tanto se haya establecido, científicamente o por métodos adecuados a las condiciones y la práctica nacionales, un vínculo directo entre la exposición a factores de riesgo que resulte de las actividades laborales y el(los) trastorno(s) mentales o del comportamiento contraído(s) por el trabajador. En lo que concierne a Venezuela, también se observa la importancia de presentar evidencias de causalidad cuando el fallo implica una consecuencia vinculante contra el patrono, tal como lo refiere Kahale (2015). Esta limitación impone que el uso del LIPT-45 y su norma de corrección logística, para la predicción tanto de niveles altos de burnout como de bienestar psicológico bajo, deben tener un valor exclusivamente preventivo o de tipo secundario, pues este tipo de intervención está "asociada a estrategias encaminadas a que tanto los trabajadores como sus supervisores puedan reconocer precozmente los signos de la mala salud mental, a fin de reducir sus consecuencias o impedir que empeoren" (Hurrel, Murpy, Sauter, \& Levi, 2001, p. 5.5). De hecho, la misma OIT reconoce la importancia que tiene la intervención secundaria para inmunizar al individuo frente a la enfermedad, comprendiendo todas aquellas intervenciones de carácter formativa (Hurrel et al., p. 5.5), lo cual se facilitaría aún más si se posee una medición regular y científicamente validada que informe la prevalencia del mobbing y su correlato sobre la salud psicológica de los trabajadores.

Desde un punto de vista legal, dicho uso es coherente con el principio de precaución que describe Lougheed (2009) y que es reconocido a nivel internacional desde la Declaración de Wingspread de 1998. . Este principio supone que "cuando una actividad genera preocupación por el daño que puede causar al medio ambiente o a la salud humana, se deben adoptar medidas precautorias incluso si una relación de causa y efecto no está establecida científicamente por completo" (Lougheed, 2009, p. A42). Y ello es así, pues no se puede prevenir, lo que no se puede predecir; por ello el uso de esta versión del LIPT-45, junto con esta norma de corrección aplicaría en el numeral c de las propuestas alternativas de prevención del acoso laboral descritas por Kahale (2015), a saber, "la fundamentación de medidas preventivas en una evaluación previa con métodos que cumplan los requisitos científicos y operativos mínimos" (p. 166). Esta afirmación es coherente con el numeral 11 de las funciones de los servicios de seguridad y salud en el trabajo, que de acuerdo con el Artículo 40 de la LOPCYMAT (2005) supone el desarrollo de programas de promoción de la seguridad y salud en el trabajo, de prevención de accidentes y enfermedades ocupacionales, de recreación, utilización del tiempo libre, descanso y turismo social. Su aplicación podría hacerse a partir de una evaluación anual tanto de las condiciones psicosociales del trabajo que mantengan igualmente una adecuada capacidad predictiva de indicadores de salud psicológica ocupacional (Millán, Calvanese \& D’Aubeterre, 2013), como del grado de percepción del mobbing en la empresa, de acuerdo con capacidad de predicción de tales indicadores de salud psicológica en el trabajo. 


\section{Conclusiones}

De los modelos obtenidos, el 4.1 para el NAQ, obtenido del AFE bajo el criterio de varianza explicada mayor al 60\% (ver tabla 3), y el H.1 [LIPT], obtenido a partir de la estructura original del test de 45 ítems de Leyman de 1996 (ver tabla 4), fueron aquellos con mejor ajuste obtenido bajo la estrategia de modelos rivales durante la fase confirmatoria del estudio. De estos, el 4.1 arrojó mayor validez de constructo, frente al H.1, por guardar un mejor ajuste con los datos obtenidos. Paradójicamente el modelo H.1 presentó mayor validez predictiva que el 4.1, al predecir mejor el bienestar psicológico y el burnout. Es así que nuestra recomendación es el uso de este último instrumento bajo la norma aquí presentada cuando se requiera hacer análisis del acoso psicológico en el trabajo en muestras venezolanas, pues como se señaló en la introducción, la validez predictiva es la estrategia que en última instancia vincula de forma indirecta a la expresión del acoso sobre la salud, dada su incapacidad para medirla directamente. Además, en términos prácticos, esta herramienta favorece el cumplimiento con lo establecido en el Instructivo del Baremo Nacional para la Asignación de Porcentaje de Discapacidad por Enfermedades Ocupacionales y Accidentes de Trabajo (2013) y la NT-02-2008 (INPSASEL, 2008), asegurando que la medición e interpretación de los resultados obtenidos tengan un debido correlato sobre indicadores de salud psicológica de los trabajadores.

En este sentido, el hecho de poseer adecuados indicadores de validez predictiva con respecto a indicadores de salud permitiría establecer, aunque sea de forma indirecta, la pertinencia del diagnóstico con este instrumento, pues, como señala Lougheed (2009), con ello se cumple al menos con el principio de precaución, el cual enfatiza que "cuando una actividad genera preocupación por el daño que puede causar al medio ambiente o a la salud humana, se deben adoptar medidas precautorias incluso si una relación de causa y efecto no está establecida científicamente por completo" (p.A42). Dicho de otra manera, se presenta al menos un indicador de correlación entre los puntajes del test y diversos indicadores de salud psicológica ocupacional.

Así mismo, se recomienda el cálculo ponderado de los puntajes obtenidos por cada sujeto en cada factor para guardar el supuesto de independencia estadística entre los factores y asegurar un mejor ajuste de la medida con respecto a los criterios de Burnout y Bienestar Psicológico General.

\section{Limitaciones}

No fue posible encontrar estadísticas oficiales actualizadas de la prevalencia de las demandas realizadas por acoso laboral en Venezuela, ni en los Tribunales, ni en el Ministerio del Poder Popular del Trabajo, ni en el INPSASEL; mucho menos de la totalidad de los instrumentos psicológicos utilizados en ellas, ni tampoco del porcentaje de sentencias ganadas y/o perdidas, lo cual dificulta la descripción de la magnitud del fenómeno en el ámbito laboral venezolano y como consecuencia, una mejor estimación del tamaño de la muestra en función a la representación en la población y los criterios metodológicos específicos para la toma de decisiones judiciales en cuanto al expertice psicológico

\section{Recomendaciones}

- Verificar la consistencia de la predictibilidad del modelo logístico de regresión encontrado en el LIPT-45 en segundas muestras (estudios confirmatorios y de predicción ad-hoc). 
- Ahondar en la direccionalidad de la relación del Factor 1 del LIPT-45 (Comprometer su salud), con respecto al burnout y a otras afecciones psicosociales en el trabajo no tan crónicas como el estrés laboral, la fatiga laboral, etc., para comprender mejor el vínculo entre éste y los indicadores de salud psicológica ocupacional.

- Utilizar las fórmulas logísticas contenidas en la tablas $\underline{9}$ o 10 para clasificar a los sujetos que poseen una alta percepción de acoso psicológico en el trabajo (que posee bajo bienestar psicológico y alto burnout) de los que tienen un nivel de percepción intermedio de acoso psicológico (que posee un bienestar psicológico bajo o un alto burnout), de los que tienen una baja percepción de acoso psicológico en el trabajo (al menos medio bienestar y al menos medio burnout), ya que ellas vinculan a los seis factores componentes del LIPT-45 con niveles específicos de bienestar psicológico y burnout.

- Indagar la relación existente entre las condiciones de trabajo y la aparición de acoso laboral.

- Indagar sobre los factores personales de los jefes y supervisores que se relacionan o influyen en la aparición de acoso laboral.

- Si bien se reconoce la inexistencia legal de una subordinación de lo mental a lo físico, se recomienda la realización de estudios enfocados en esclarecer los efectos físicos y sociales del acoso laboral, en especial indicadores inmunológicos.

\section{Referencias}

Anastasi, A. \& Urbina, S. (1998). Test Psicológicos. México, D.F., México: Editorial Prentice Hall. https://books.google.com.pe/books/about/Tests psicol\%C3\%B3gicos. html?id=FV01zgFukOcC

Anderson, J., \& Gerbing, D. (1988). Structural equation modeling in practice: a review and recommended two-step approach. Psychological Bulletin, 103(3), 411-423. Recuperado de: http://psycnet.apa.org/psycinfo/1989-14190-001

Arnau, J. (1979). Psicología Experimental: Un enfoque metodológico. México, D.F., México: Editorial Trillas. http://www.iberlibro.com/buscar-libro/titulo/psicolog\%EDaexperimental-enfoque-metodol\%F3gico/

Batista-Foguet, J., Coenders, G., \& Alonso, J. (2004). Análisis factorial confirmatorio. Su utilidad en la validación de cuestionarios relacionados con la salud. Medicina Clínica, 122(1), 21-27. Recuperado de: http://www.elsevier.es/es-revista-medicina-clinica-2-articulo-analisis-factorial-confirmatorio-su-utilidad-13057542

Blanch, J., Sahagún, M., \& Cervantes, G. (2010). Estructura Factorial del Cuestionario de Condiciones de Trabajo. Revista de Psicología del Trabajo y de las Organizaciones, 26(3), 175-189. Recuperado de: http://www.copmadrid.org/webcopm/resource. do? recurso $=3000 \&$ numero $=20101223115448093000$

Boomsma, A. (2000). Reporting Analyses of Covariance Structures. Structural Equation Modeling, 7(3), 461-483. Recuperado de: http://www.gmw.rug.nl/ boomsma/ boomsma00.pdf

Borsboom, D. (2006). The attack of the psychometricians. Psychometrika, 71(3), 425440. Recuperado de: http://www.ncbi.nlm.nih.gov/pmc/articles/PMC2779444/

Bunge, M. (1966). La ciencia, su método y su filosofía. Buenos Aires, Argentina: Editorial Siglo XXI. https://bibliophiliaparana.wordpress.com/2011/05/18/bungemario-la-ciencia-su-metodo-y-su-filosofial

Cardozo, G., \& López, C. (2009). Situación de mobbing en una institución de salud pública ubicada en Valencia, estado Carabobo: una realidad que subyace en este ámbito laboral [Tesis de pregrado inédita]. Escuela de Relaciones Industriales. Universidad de Carabobo, Bárbula, Venezuela. 
Código Orgánico Procesal Penal. (2012, 12 de junio). Gaceta Oficial de la República Bolivariana de Venezuela [Extraordinaria], 6.078, junio 15, 1999. http://www.oas. org/juridico/PDFs/mesicic4 ven cod org proc penal.pdf

Constitución de la República Bolivariana de Venezuela. (1999, 20 de diciembre). Gaceta Oficial de la República Bolivariana de Venezuela, 36.860, diciembre 30, 1999. http://pdba.georgetown.edu/Constitutions/Venezuela/ven1999.html

Delgado, R. (2015). Las pruebas en el Proceso Penal Venezolano (6 ed.). Caracas, Venezuela: Vadell Hermanos Editores. http://www.probatorio.org/biblografia/

Devis-Echandía, H. (1993). Teoría general de la prueba judicial ( $4^{a}$ ed.). Medellín, Colombia: Editorial Diké. http://www.probatorio.org/biblografia/

Ege, H. (2002). Mobbing. New Perspectives and results from an italian investigation. Bologna, Italia. Pitagora Editrice. http://www.pitagoragroup.it/pited/Ege\%20inglese.html

Einarsen, S., Matthiesen, S.B., \& Skogstad, A. (1998). Bullying, burnout and well-being among assistant nurses. Journal of Occupational Health and Safety - Australia and New Zealand, 14, 263-268. Recuperado de: https://www.researchgate.net/publication/275340145 Bullying burnout and well-being among assistant nurses

Einarsen, S., \& Hoel, H. (2001). The Negative Acts Questionnaire: Development, validation and revision of a measure of bullying at work. Trabajo presentado en el $10^{\circ}$ European Congress on Work and Organizational Psychology, Praga, República Checa. $\quad$ http://www.scielo.org.co/scieloOrg/php/reference. php?pid=S1657$92672008000200003 \&$ caller=www.scielo.org.co\&lang $=e s$

Einarsen, S., \& Hoel, H. (2006). Measuring bullying and harassment in the workplace. Development and validity of the revised Negative Acts Questionaire. A manual. Bergen, Noruega: Universidad de Bergen. https://www.researchgate.net/publication/228079665 Measuring exposure to bullying and harassment at work Validity factor structure and psychometric properties of the Negative Acts Questionnaire-Revised

Einarsen, S., \& Raknes, B.I. (1997). Harassment in the workplace and the victimization of men. Violence and Victims, 12(1), 247-263. Recuperado de: http://www. ncbi.nlm.nih.gov/pubmed/9477540

Einarsen, S., Hoel, H., \& Notelaers, G. (2009). Measuring exposure to bullying and harassment at work: Validity, factor structure and psychometric properties of the Negative Acts Questionnaire- Revised. Work \& Stress, 23(1), 24-44. Recuperado de: http://www.tandfonline.com/doi/abs/10.1080/02678370902815673

Einarsen, S., Hoel, H., Zapf, D., \& Cooper, C. (Eds.). (2003). Bullying and emotional abuse in the workplace International perspectives in research and practice (pp. 3-30). London: Taylor and Francis. Recuperado de: https://www.researchgate. net/file.PostFileLoader.html?id=5613552d6307d93e378b45df\&assetKey=AS\% 3A281421676597248\%401444107564277

Escartín, J., Rodríguez-Carballeira, Á., Gómez-Benito, J., \& Zapf, D. (2010). Development and validation of the workplace bullying scale EAPA-T. International Journal of Clinical and Health Psychology, 10(3), 519-539. http://www.ub.edu/grupvp/index.php/es/47-publicaciones/mobbing/99-development-and-validation-of-theworkplace-bullying-scale-eapa-t.html

Federación de Psicólogos de Venezuela. (1981). Código de ética profesional. Recuperado de: http://fpv.org.ve/documentos/codigodeetica.pdf

Fidalgo, Á., \& Piñuel, I. (2004). La escala Cisneros como herramienta de valoración del mobbing. Psicothema, 16(4), 615-624. Recuperado de: http://www.psicothe-

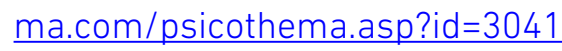


Figueiredo-Ferraz H., Gil-Monte P. R., Grau-Alberola E., Llorca-Pellicer M., \& GarcíaJuesas J. A. (2012). Influence of some psychosocial factors on mobbing and its consequences among employees working with people with intellectual disabilities. Journal of Applied Research in Intellectual Disabilities, 25(1), 455-63. Recuperado de: http://www.ncbi.nlm.nih.gov/pubmed/22890946

Figueiredo-Ferraz H., Gil-Monte P. R., \& Olivares-Faúndez, V. E. (2015). Influence of mobbing (workplace bullying) on depressive symptoms: a longitudinal study among employees working with people with intellectual disabilities. Journal of Intellectual Disability Research, 59(1), 39-47. Recuperado de: http://onlinelibrary. wiley.com/doi/10.1111/jir.12084/abstract

Fornés, J., Martínez-Abascal, M., \& García, G. (2008). Análisis factorial del Cuestionario de Hostigamiento Psicológico en el trabajo en profesionales de Enfermería. International Journal of Clinical and Health Psychology, 8(1), 267-283. Recuperado de: http://www.redalyc.org/articulo.oa?id $=33780118$

Furham, A. (1995). Personalidad y diferencias individuales en el trabajo. Pirámide, Madrid: España http://www.casadellibro.com/libro-personalidad-y-diferenciasindividuales-en-el-trabajo/9788436808506/493049

García-Izquierdo, M., Llor, B., Sáez, M. C., Ruiz, J. A., Blanco, J. R., \& Campillo, M. J. (2004). Evaluación del acoso psicológico en el trabajo: El NAQ-RE. Revisión de la adaptación española. Comunicación oral presentada al VII European Conference on Psychological Assessment, Málaga. España. http://www.masterforense. com/pdf/2013/2013art1.pdf

García-Izquierdo, M., Sáez, M. C., \& Llor, B. (2003). Validación de la escala NAQ de Einarsen y Raknes. (1997). Comunicación oral presentada al VIII Congreso Nacional de Psicología Social, Málaga. España. http://www.masterforense.com/ pdf/2013/2013art1.pdf

Gil-Monte P. R., Carretero N., \& Luciano J. V. (2006) Prevalencia del mobbing en trabajadores de centros de asistencia a personas con discapacidad. Revista de Psicología del Trabajo y de las Organizaciones, 2(1), 275-291. Recuperado de: http:// www.redalyc.org/articulo.oa?id $=231317121003$

Giorgi, G., Matthiesen, S. B., \& Einarsen, S. (2006, June). Italian validation of the Negative Acts Questionnaire. Trabajo presentado a la 5tb International Conference on Bullying and Harassment in the Workplace, Dublín, Irlanda. https://www.uam. es/gruposinv/esalud/Articulos/Salud\%20Laboral/Assessing-workplace-bullying.pdf

Glaso, L., Matthiesen, S. B., Nielsen, M. B., \& Einarsen, S. (2007). Do targets of workplace bullying portray a general victim personality profile? Scandinavian Journal of psychology, 48(4), 313-319. Recuperado de: http://www.ncbi.nlm.nih.gov/ pubmed/17669221

Gómez-Restrepo, C., \& Delgado, M. (2006). Apuntes sobre causalidad. Revista Colombiana de Psiquiatría, 35(1), 96 - 104. Recuperado de: http://www.redalyc.org/ pdf/806/80635109.pdf

González de Rivera, J. L., \& Rodríguez-Abuín, M. J. (2003). Cuestionario de estrategias de acoso psicológico: el LIPT-60. Psiquis, 24(2), 59-66. Recuperado de: http:// www.psicoter.es/component/option,com docman/Itemid,73/gid,153/task,doc download/

Gonzalez de Rivera, J. L., \& Rodríguez-Abuín, M. J. (2005) Cuestionario de estrategias de acoso en el trabajo. El LIPT-60. Editorial EOS: Madrid, España. http://www.psicoter.es/pdf/manual-lipt60.pdf

González, D., \& Graña, J. (2009). El acoso psicológico en el lugar de trabajo: prevalencia y análisis descriptivo de una muestra multiocupacional. Psicothema, 21(2), 288293. Recuperado de: http://www.psicothema.com/psicothema.asp?id=3628 
Hair, J., Anderson, R., Tatham, R., \& Black, W. (1999). Análisis Multivariante (5ta. ed.). Madrid: Prentice Hall. www.cosaslibres.com/search/pdf/analisis-multivariante-hair/10

Hauge, L. J., Skogstad, A., \& Einarsen, S. (2007). Relationships between stressful work environments and bullying. Results of a large representative study. Work \& Stress, 21(3), 220-242. Recuperado de: http://www.tandfonline.com/doi/ abs/10.1080/02678370701705810

Hayton, J.C., Allen, D.G., \& Scarpello, V. (2004). Factor retention decisions in exploratory factor analysis: A tutorial on parallel analysis. Organizational Research Methods, 7, 191-205. Recuperado de: http://orm.sagepub.com/content/7/2/191. abstract

Hernández, R., Fernández, C., \& Baptista, P. (4ta Ed.) (2007). Metodología de la Investigación. México: Mc Graw Hill. https://es.scribd.com/doc/36909622/Metodologiade-La-Investigacion-4ta-Edicion-Sampieri-2006

Hoel, H., \& Cooper, C. (2000a). Workplace bullying in Britain. Employee Health Bulletin, 14, 9-14. http://www.scielo.br/pdf/pe/v12n3/v12n3a02.pdf

Hoel, H., \& Cooper, C. (2000b). Destruptive conflict and bullying at work. British Occupational Health Research Foundation, Reino Unido: Manchester School of Management, University of Manchester Institute Science and Technology. Recuperado de: http://www.adapttech.it/old/files/document/19764Destructiveconfl.pdf

Hoel, H., Cooper, C. L., \& Faragher, B. (2001). The experience of bullying in Great Britain: The impact of organizational status. European Journal of Work and Organizational Psychology, 10(4), 443-465. Recuperado de: http://www.tandfonline.com/ doi/abs/10.1080/13594320143000780?journalCode=pewo20

Hoel, H., Faragher, B., \& Cooper, C. (2004). Bullying is detrimental to health, but all bullying behaviours are not necessarily equally damaging. British Journal of Guidance and Counselling, 32(3), 367-387. Recuperado de: http://www.tandfonline.com/doi/abs/10.1080/03069880410001723594

Hu, L., \& Bentler, P. M. (1998). Fit indices in covariance structure modeling: Sensitivity to underparameterized model misspecification. Psychological Methods, 3, 424-453. Recuperado de: http://psycnet.apa.org/index.cfm?fa=buy. optionToBuy\&id=1998-11538-003

Hurrel, J., Murpy, L., Sauter, S., \& Levi, L. (2001). Salud Mental [Capitulo 5]. En Ministerio de Trabajo y Asuntos Sociales de España (Eds). Enciclopedia de Salud y Seguridad en el Trabajo de la Organización Internacional del Trabajo. Recuperado de: http://www.mtas.es/insht/EncOlT/Index.htm

Instituto Nacional de Estadística (INE). (2012). Situación en la Fuerza de Trabajo de Venezuela. Informe Semestral. (2do. Semestre del 2011). Recuperado de: http:// www.ine.gov.ve/documentos/Social/FuerzadeTrabajo/pdf/Informesemestral. $\underline{\mathrm{pdf}}$

Instituto Nacional de Prevención, Salud y Seguridad Laborales (INPSASEL). (2008). Norma Técnica para la Declaración de Enfermedad Ocupacional (NT-02-2008). Disponible en: http://www.inpsasel.gob.ve/moo doc/Nor_DeclEnfer_Ocup.pdf

Instituto Nacional de Prevención, Salud y Seguridad Laborales (INPSASEL). (2013). Instructivo del Baremo Nacional para la Asignación de Porcentaje de Discapacidad por Enfermedades Ocupacionales y Accidentes de Trabajo. Gaceta Oficial de la República Bolivariana de Venezuela, 40154, abril 25, 2013. biblioteca.ceditel.gob. ve/ABCD/bases/biblo/texto/40154.pdf

Jiménez, E. (2010). Propiedades psicométricas del Leymann Inventory of Psychological Terrorization (LIPT-60). Gestión y Gerencia, 4(1), 61-82. Recuperado de: http:// www.ucla.edu.ve/DAC/investigacion/gyg/GyG\%202010/Abril\%202010/4-\%20 Esther\%20 Jimenez.pdf 
Kahale, D. (2015). El acoso laboral (mobbing): Tratamiento jurídico y preventivo (2ª). Caracas, Venezuela: Vandell hermanos editores.

León-Pérez, J. M., Notelaers, G., Arenas, A., Munduate, L., \& Medina, F. (2014). Identifying Victims of Workplace Bullying by Integrating Traditional Estimation Approaches into a Latent Class Cluster Model. Journal of Interpersonal Violence, 29(7), 1155 1177. Recuperado de: http://www.ncbi.nlm.nih.gov/pubmed/24257593

Ley Orgánica de Prevención, Condiciones y Medio Ambiente de Trabajo (LOPCYMAT). (2005, 25 de julio). Gaceta Oficial de la República Bolivariana de Venezuela, 38.236, julio 26, 2005. http://www.inpsasel.gob.ve/moo news/lopcymat.html

Ley Orgánica del Trabajo, las Trabajadoras y los Trabajadores (LOTTT). (2012, 7 de mayo). Gaceta Oficial de la República Bolivariana de Venezuela [Extraordinaria], 6.076, abril 30, 2012. www.lottt.gob.ve/wp-content/uploads/2012/05/LOTTT-Gaceta-6.076.pdf

Ley Orgánica de la Salud (LOS). (1998, 26 de agosto). Gaceta Oficial de la República de Venezuela, 36.579, noviembre 11, 1998. www.fundaribas.gob.ve/paginaweb/ pdf/salud.pdf

Leymann, H. (1990). Mobbing and psychological terror at workplaces. Violence and Victims, 5, 119-126. Recuperado de: http://www.mobbingportal.com/ LeymannV\%26V1990(3).pdf

Lougheed, T. (2009). Mirando Hacia Adentro. Ciencia \& Trabajo, 11(32), A37 - A43. Recuperado de: http://www.cienciaytrabajo.cl/cyt/EdicionesAnteriores/Volumen\%2032.pdf

Marsh, H.W., Hau, K. T., \& Wen, Z. (2004). In search of golden rules: Comment on hypothesis-testing approaches to setting cutoff values for fit indexes and dangers in overgeneralizing Hu and Bentler's (1999) findings. Structural Equation Modeling, 11, 320-341. Recuperado de: http://www.tandfonline.com/doi/abs/10.1207/ s15328007sem1103 2

Matthiesen, S.B., \& Einarsen, S. (2001). MMPI-2 configurations among victims of bullying at work. European Journal of Work and Organizational Psychology, 32, 335356. Recuperado de: http://folk.uib.no/pspsm/documents/Matthiesen og Einarsen-MMPI-2 and bullying 2001.pdf

Matthiesen, S. B., \& Einarsen, S. (2007). Perpetrators and targets of bullying at work: Rolestress and individual differences. Violence and Victims, 22(6), 735-753. Recuperado de: http://folk.uib.no//pspsm/documents/Bullying-Matthiesen-Einarsen-2007.pdf

Matthiesen, G. E., Einarsen, S., \& Mykletun, R. (2008). The occurrences and correlates of bullying and harassment in the restaurant sector. Scandinavian Journal of Psychology, 49, 59-68. Recuperado de: http://www.ncbi.nlm.nih.gov/ pubmed/18190403

Mayhew, C., \& Chappell, D. (2007). Workplace violence: An overview of patterns of risk and the emotional/stress consequences on targets. Internationl Journal of Law and Psychiatry, 30, 327-339. Recuperado de: http://www.ncbi.nlm.nih.gov/ pubmed/17628681

Mikkelsen. E.G., \& Einarsen, S. (2001). Bullying in Danish worklife: Prevalence and health correlates. European Journal of Work and Organisational Psychology, 10, 393-413. Recuperado de: http://www.tandfonline.com/doi/abs/10.1080/13594 320143000816?journalCode=pewo20

Mikkelsen, E. G., \& Einarsen, S. (2002). Basic assumptions and symptoms of posttraumatic stress among victims of bullying at work. European Journal of Work and Organizational Psychology, 77(1), 87-111. Google books 
Millán, A., \& D' Aubeterre, M. E. (2011). Validación de la Escala de Bienestar Psicológico en una muestra multiocupacional venezolana. Revista CES Psicología, 4(1), 52-71. Recuperado de http://dialnet.unirioja.es/servlet/fichero articulo? codigo $=3672741 \&$ orden $=0$

Millán, A., \& D'Aubeterre, M. E. (2012). Propiedades psicométricas del Maslach Burnout Inventory-GS en una muestra multiocupacional venezolana. Revista de Psicología, 30(1), 104-128. Recuperado de http://revistas.pucp.edu.pe/index.php/ psicologia/article/view/2627/2574

Millán, A., Calvanese, N., \& D'Aubeterre, M. E. (2013). Propiedades psicométricas del cuestionario de condiciones de trabajo (qCT) en una muestra multiocupacional venezolana. Revista CES Psicología, 6(2), 28-52. Disponible en: http://revistas. ces.edu.co/index.php/psicologia

Ministerio del Poder Popular de Ciencia, Tecnología e Innovación (MCTI). (2011). Necesidades de Investigación 2011. Recuperado de: http://www.ucv.ve/fileadmin/user upload/facultad humanidades/documentos/coordinacion de investigacion/2011/31.01.2011 necesidades investigacion mppctii 1 .pdf

Montero, I., \& León, O. (2007). Guía para nombrar los estudios de investigación en psicología. International Journal of Clinical and Health Psychology, 7(3), 847-862. Recuperado de: http://www.aepc.es/ijchp/GNEIP07_es.pdf

Moreno-Jiménez, B., Rodríguez-Muñoz, A., Martínez-Gamarra, M., \& Gálvez, M. (2007). Assessing workplace bullying: Spanish validation of a reduced versión of Negative Acts Questionnaire. The Spanish Journal of Psychology, 10(2), 449-457. Recuperado de: http://www.ncbi.nlm.nih.gov/pubmed/17992971

Moayed, F., Daraiseh, N., Shell, R., \& Salem, S. (2006). Workplace bullying: A systematic review of risk factors and outcomes. Theoretical Issues in Ergonomics Science, 7(1), 311-327. Recuperado de: http://www.tandfonline.com/doi/ abs/10.1080/14639220500090604

Niedl, K. (1996). Mobbing and well-being: Economic and personnel development implications. European Journal of Work and Organizational Psychology, 5(2), 239-249. Recuperado de: http://www.tandfonline.com/doi/ abs/10.1080/13594329608414857

Nielsen, M. B., \& Einarsen, S. (2008). Sampling in research on interpersonal aggression. Aggressive Behavior, 34(3), 265-272. Recuperado de: http://www.ncbi.nlm. nih.gov/pubmed/17786923

Notelaers, G., Einarsen, S., De Witte, H., \& Vermunt, J. (2006). Measuring exposure to bullying at at work: The validity and advantages of the latent class cluster approach. Work \& Stress, 20(4), 288-301. Recuperado de: http://www.tandfonline. com/doi/abs/10.1080/02678370601071594

Nunally, J., \& Bernstein, I. (1995). Teoría psicométrica (2da Ed.). México, D.F.: México, Mc Graw Hill.

Oramas, A., González, A., \& Vergara, A. (2007). El desgaste profesional: evaluación y factorialización del MBI-GS. Revista Cubana de Salud y Trabajo, 8(1), 37-45. Recuperado de: http://bvs.sld.cu/revistas/rst/vol8 1 07/rst06107.html

Organización Internacional del Trabajo (OIT). (2010). Lista de enfermedades profesionales (revisada en 2010). Serie Seguridad y Salud en el Trabajo N 74, Ginebra: Suiza. Recuperado de: http://www.ilo.org/safework/info/publications/ WCMS 150327/lang--es/index.htm

Organización Mundial de la Salud (OIT).(2010). Ambientes de Trabajo Saludables:un modelo para la acción para empleadores, trabajadores, autoridades normativas y profesionales. Recuperado de: http://www.who.int/iris/handle/10665/44317\#sthash. BtUVWfYg.dpuf 
Papalia, D., \& Olds, S. (1997). Desarrollo Humano (6ta ed.). México: McGraw-Hill.

Pardo, A., \& Ruiz, M. (2002). SPSS 11: Guía para el análisis de datos. Madrid, España: McGraw Hill.

Pérez-Gill, J., Chacón, S., \& Moreno, R. (2000). Validez de constructo: el uso de análisis factorial exploratorio-confirmatorio para obtener evidencias de validez. Psicothema, 12(2), 442-446. Recuperado de: http://www.psicothema.com/pdf/601. $\underline{\mathrm{pdf}}$

Piñuel, I. (2001). Mobbing. La lenta y silenciosa alternativa al despido. AEDIPE, 17, 1955. Recuperado de: http://www.mobbing.nu/pinnuel-2003-lalentaysilenciosa.rtf

Ponsoda, V. (2009). Metodología al servicio del psicólogo. Papeles del Psicólogo, 31(1), 2-6. Recuperado de: http://www.papelesdelpsicologo.es/pdf/1791.pdf

Prieto, G., \& Muñiz, J. (2000). Un modelo para evaluar la calidad de los test utilizados en España. Papeles del Psicólogo, 77(1), 65-72. Recuperado de: http://www.redalyc.org/articulo.oa?id=77807709

Sánchez-Cánovas, J. (2007). Manual Escala de Bienestar Psicológico (2ª). Madrid: TEA Ediciones, S.A. Google books

Seijas, F. (2003). Investigación electoral: encuestas electorales (2da. ed.). Caracas, Venezuela: Ediciones del Rectorado - Universidad Central de Venezuela. http://www. scielo.org.pe/scielo.php?pid=S0254-92472012000100005\&script=sci arttext

Vartia, M. (1996). The sources of bullying - psychological work environment and organizational climate. European Journal of Work and Organizational Psychology, 5(2), 203-214. Recuperado de: http://www.tandfonline.com/doi/ abs/10.1080/13594329608414855

Vie, T. L., Glaso, L., \& Einarsen, S. (2011). Health outcomes and self-labeling as a victim of workplace bullying. Journal of Psychosomatic Research, 70, 37-43. Recuperado de: http://www.ncbi.nlm.nih.gov/pubmed/21193099

World Health Organization (WHO). (1946). Official record of the World Health Organitation N²: Proceedings and final acts of the International Health Conference, New York: United States. Recuperado de: http://apps.who.int/iris/bitstream/10665/85573/1/0fficial record2 eng.pdf

Zapf, D. (1999). Organizational, work group related and personal causes of mobbying/bullying at work. International Journal of Manpower, 20(1), 70-85. Recuperado de: http://www.emeraldinsight.com/doi/abs/10.1108/01437729910268669

Zapf, D., Knorz, C., \& Kulla, M. (1996). On the relationships between mobbing factors, and job content, the social work environment and health outcomes. European Journal of Work and Organizational Psychology, 5, 215-237. Recuperado de: http:// www.tandfonline.com/doi/abs/10.1080/13594329608414856 